\title{
Experimental and Theoretical Investigations of Free Radical Photopolymerization: Inhibition and Termination Reactions
}

Julien Christmann, Christian Ley, Xavier Allonas, Ahmad Ibrahim, Céline Croutxé-Barghorn Laboratory of Macromolecular Photochemistry and Engineering, 3b rue Alfred Werner, 68093 Mulhouse, France

${ }^{*}$ Corresponding author

E-mail address: christian.ley@uha.fr (C. Ley)

\begin{abstract}
In this work, the inhibition and termination reactions occurring throughout a free radical photopolymerization initiated by a type-I photoinitiator are studied by kinetic modeling. The role of the macroradicals as the main oxygen trapping agents during the inhibition time is identified, and the absence of primary radical consumption by oxygen can be related to a high initiation efficiency at early times. The ratio of the termination reactions reveals that bimolecular termination remains the principal pathway for the cessation of macromolecule growth, even at high polymer conversion. Moreover, the evolution of the termination ratio during the polymerization can be correlated to both the diffusional control of the polymerization reactions as the polymer network grows and the photoinitiator consumption. Finally, the effect of the incident light intensity and the initial photoinitiator concentration on the termination reactions is assessed, and the validity of the steady-state assumption applied to the macroradical concentration discussed.
\end{abstract}

\section{Keywords}

Radical photopolymerization

Kinetic modeling

Type-I photoinitiator

Oxygen inhibition

Termination reactions

Steady-state assumption 


\section{Introduction}

Photopolymerization is nowadays a high-performance technology for the synthesis of polymer materials in various industrial fields such as coatings (paints, inks, varnishes...), dental resins, automotive, holographic data storage, stereolithography, etc. Several advantages over the thermally-induced polymerization process make photopolymerization an eco-friendly technology, with time, money and energy savings, waste reduction, absence of solvent, spatio-temporal control of the reaction, etc. [1-4]. The photopolymerization process basically consists in the conversion of a liquid monomer mixture into a solid three-dimensional polymer network under light irradiation. Depending on the photogenerated initiating species, different types of photopolymerization processes can be distinguished: radical, cationic and anionic. Among them, free radical photopolymerization (FRP) remains the main process currently implemented [5].

The FRP mechanism consists in a complex combination of reactions which can be divided into three main steps: photoinitiation, propagation and termination [2,3,6-9]. During the photoinitiation, initiating radicals $R^{*}$ are produced under light exposure. A photoinitiating system (PIS) is thus generally required to convert photons into such a chemical potential [2,10-13]. Type-I PIS rely on the bond dissociation of a photoinitiator molecule to yield one (or two) initiating radical(s), while type-II systems associate a photoinitiator with a coinitiator to insure the production of active species. Photoproduced radicals are then able to react onto monomer $\mathrm{C}=\mathrm{C}$ double bonds and generate chain-initiating radicals $\mathrm{R}(\mathrm{C}=\mathrm{C})_{1}$. Macroradicals $\mathrm{R}(\mathrm{C}=\mathrm{C})_{\mathrm{n}}$. grow during the propagation step, through successive additions of monomer units associated to the regeneration of the active radical center after each addition. Termination reactions finally stop the growth of the macromolecules, either by bimolecular (combination, disproportion [69] or primary radical termination PRT [9,14-17]) or monomolecular (radical trapping by occlusion $[3,9,18-30])$ mechanisms. All termination modes should occur simultaneously but the proportion of each of them is expected to evolve during the polymerization, because of the progressive increase of the medium viscosity which limits the species motion as the tridimensional network develops. At low and medium conversion, bimolecular termination is supposed to prevail, while radical trapping by occlusion should be the major termination way when the reaction medium becomes vitrified. Nevertheless, there are only a few studies in the literature dealing with the evolution of the termination modes during the FRP process [9]. Radical photopolymerization is also known to be strongly inhibited by molecular oxygen, either by its reaction with the excited state of the photoinitiator or the (macro)radicals to yield inactive peroxy radicals $\mathrm{ROO}^{\circ}$ and $\mathrm{R}(\mathrm{C}=\mathrm{C})_{n} \mathrm{OO} \cdot[2-10,25,31-33]$. However, the distinction between the inhibition reactions has only been scarcely addressed in a few papers [34,35]. 
The progressive increase of the medium viscosity and the diffusional control of the elementary reactions mainly contribute to the complexity of the (photo)polymerization process, with effects such as the autoacceleration (increase of the polymerization rate $R_{p}$ when bimolecular termination becomes restricted) and the autodeceleration (decrease of $R_{p}$ when propagation also becomes diffusion-controlled) $[3,6-8,36]$. Reaction diffusion termination is also specific to this strong increase of viscosity. Indeed, immobilized macroradicals are able to pursue their growth through the addition of small monomer units by propagation, until their chain-ends come together and react bimolecularly (by combination or disproportion) [3,37-53]. The corresponding bimolecular termination rate constant $\mathrm{k}_{\mathrm{t}, \mathrm{b}}$ is then proportional to the propagation rate constant $k_{p}$ with a reaction diffusion factor $R_{r d}$ (Eq. 1). Reaction diffusion termination starts as soon as diffusional bimolecular termination is hindered by the increase of the medium viscosity.

$\mathrm{k}_{\mathrm{t}, \mathrm{b}}=\mathrm{R}_{\mathrm{rd}} \mathrm{k}_{\mathrm{p}}[\mathrm{C}=\mathrm{C}]$

Better understanding of the complex FRP mechanism and its related effects is a recurrent topic in polymer science. For that purpose, several modeling methods have been developed, starting with the statistical approach developed by Flory in the 1940's [54-57] and followed by spatial [58-62] and kinetic modeling [14-17,28-30,38,43,44,46,47,63-84]. This last method appears especially interesting as it: $i$. enables direct comparison between experimental and simulated polymerization kinetics, ii. is relatively simple to implement, iii. gives access to the concentration of species which are difficult or even impossible to obtain from experiment (macroradicals, initiating radicals...). The one developed by Bowman's group is currently the most complete, considering the whole photopolymerization process from light absorption by a type-I PIS to termination reactions [14,46,47,78-81]. It has been notably used to determine the effect of oxygen inhibition on the propagation and termination rate constants [82], as well as model layer-by-layer photopolymerization [83] by Taki et al.. Such a kinetic model has been recently adapted for the photoinitiation involving a type-II PIS combining isopropylthioxanthone and triazine derivatives, evidencing the crucial role of back electron transfer in the kinetics of three-dimensional polymer network formation [84]. However, there is still a lack of quantitative data concerning the amount and the competition between the termination reactions for such a simple system as a type-I photoinitator. Indeed, among the photopolymerization kinetic models available in the literature, most of them does not take into account simultaneously all the termination pathways [16,17,29-30,38,43,44,46,47,64-79,8183] and/or does not address their evolution all along the photopolymerization process $[16,17,38,63-74,80-83]$. 
This paper aims to improve the understanding of the crucial oxygen inhibition and termination steps through the modeling of the whole FRP process initiated by a type-I photoinitiator. A kinetic model considering simultaneously all the termination pathways is thus optimized by comparison with experimental photopolymerization kinetics. It is subsequently modified to identify the main oxygen trapping agent in the case of a laminated photopolymerization reaction, and discuss the dual role of the initiating radicals between initiation and termination in function of their reactivity towards the monomer and the macroradicals as the diffusion control settles. For the first time, the evolution of each of the termination modes is discussed with numerical values all along the photopolymerization reaction in conjunction with the evolution of the polymerization rate constants and the concentration of the reactive species. This study is finally extended to different incident light intensities and photoinitiator initial concentrations, and a new view on the validity of the pseudo-steady-state assumption is provided in relation with the advancement of the polymerization reaction.

\section{Experimental section}

\subsection{Materials}

Type-I PIS diphenyl(2,4,6-trimethylbenzoyl)phosphine oxide (TPO) was purchased from BASF. Diacrylic monomer ethoxylated bisphenol A diacrylate (SR349) was supplied by Sartomer and dimethylsulfoxide (DMSO, $\geq 99.8 \%$ ) by Sigma-Aldrich. Their structures are given in the ESI (Figure S1). All compounds were used as received.

\subsection{Experimental methods}

The formulations for the experimental kinetic analyses were based on a mixture of SR349 (90 wt\%) and DMSO (10 wt\%), in which 0.5 to 1 wt \% of TPO has been dissolved. They were stirred overnight and kept in the dark. The photopolymerization kinetics were experimentally followed with a real-time Fourier transform infrared (RT-FTIR) spectrometer Vertex 70 from Brucker Optics, equipped with a nitrogen liquid-cooled mercury cadmium telluride (MCT) detector [85]. It operated in rapid scan mode with a sampling interval of $0.12 \mathrm{~s}$ and $4 \mathrm{~cm}^{-1}$ resolution. The light irradiation was provided by a $395 \mathrm{~nm}$ LED (Roithner, LaserTechnik) whose incident intensity was checked with a calibrated fiber optic spectrometer (Ocean Optics, USB4000), on which a polypropylene (PP) film and a $\mathrm{CaF}_{2}$ pellet were placed to take into account light loss on the interfaces. Oxygen diffusion from the atmosphere was avoided by performing laminated experiments (the photosensitive resin was placed between two PP films and two $\mathrm{CaF}_{2}$ pellets). A $25 \mu \mathrm{m}$ Teflon spacer was also used in order to adjust the thickness of the formulation. 
The FRP kinetics were measured by following in real time the disappearance of the $\mathrm{C}=\mathrm{C}$ bond stretching vibration band of the diacryclic monomer at $1637 \mathrm{~cm}^{-1}$. The degree of conversion was calculated as:

$\operatorname{Conv}(\%)=\frac{\left(\mathrm{A}_{1637}\right)_{0}-\left(\mathrm{A}_{1637}\right)_{\mathrm{t}}}{\left(\mathrm{A}_{1637}\right)_{0}} \times 100$

where $\left(A_{1637}\right)_{0}$ and $\left(A_{1637}\right)_{t}$ are, respectively, the areas of the $1637 \mathrm{~cm}^{-1}$ vibration band before light irradiation and at an irradiation time $t$. The rate of polymerization $R_{p}$ is derived from the degree of conversion by:

$\mathrm{R}_{\mathrm{p}}=\mathrm{k}_{\mathrm{p}}\left[\mathrm{R}(\mathrm{C}=\mathrm{C})_{\mathrm{n}}{ }^{\bullet}\right][\mathrm{C}=\mathrm{C}]=\frac{\mathrm{d} \operatorname{Conv}(\%)}{\mathrm{dt}} \times \frac{[\mathrm{C}=\mathrm{C}]_{0}}{100}$

with $[\mathrm{C}=\mathrm{C}]_{0}$ the initial concentration of acrylic doubles bonds in the formulation. Each experiment was repeated three times to ensure a good reproducibility and the data presented here result from an average.

\subsection{Computational methods}

Kinetic modeling was implemented in Wolfram Mathematica 9 software. The rate constants were defined as functions of the fractional free volume $v_{f}$, and the reactions of the photopolymerization mechanism were expressed as a set of differential equations, which was numerically solved with the NDSolve function [84]. Conversion was directly calculated with the values of the acrylic double bond concentration $[\mathrm{C}=\mathrm{C}]$ at zero and t time (Eq. 4) and $\mathrm{R}_{\mathrm{p}}$ was calculated after numerical resolution with Eq. 3.

$\operatorname{Conv}(\%)=\frac{[\mathrm{C}=\mathrm{C}]_{0}-[\mathrm{C}=\mathrm{C}]_{\mathrm{t}}}{[\mathrm{C}=\mathrm{C}]_{0}} \times 100$

\section{Results and discussion}

\subsection{Kinetic model conception}

The main kinetic rate constants are defined as functions of the fractional free volume $v_{f}$ in order to take into account their progressive diffusional control throughout the photopolymerization process [14,46,47,78-81]. $v_{f}$ represents the fraction of unoccupied volume in the reaction medium. It decreases as the polymerization proceeds according to:

$$
\begin{aligned}
& v_{\mathrm{f}}=0.025+\alpha_{\mathrm{M}}\left(\mathrm{T}-\mathrm{T}_{\mathrm{g}, \mathrm{M}}\right) \Phi_{\mathrm{M}}+\alpha_{\mathrm{P}}\left(\mathrm{T}-\mathrm{T}_{\mathrm{g}, \mathrm{P}}\right)\left(1-\Phi_{\mathrm{M}}\right) \\
& \Phi_{\mathrm{M}}=\frac{1-\operatorname{Conv}}{1-\operatorname{Conv}+\operatorname{Conv} \times\left({ }^{\left.\rho_{\mathrm{M}} / \rho_{\mathrm{P}}\right)}\right)}
\end{aligned}
$$


Subscripts $\mathrm{M}$ and $\mathrm{P}$ refer to monomer and polymer respectively, with $\alpha$ the thermal expansion coefficient (difference between liquid and glassy state expansion coefficients), $T_{g}$ the glass transition temperature, $\Phi$ the volume fraction and $\rho$ the volumetric mass density.

The expressions for the propagation $\left(\mathrm{k}_{\mathrm{p}}\right)$ and bimolecular termination $\left(\mathrm{k}_{\mathrm{t}, \mathrm{b}}\right)$ rate constants are given in Eqs. 7 and 8 [14,46,47,78-81]. The expression of $k_{t, b}$ includes both diffusional bimolecular termination and subsequent reaction diffusion processes. $\mathrm{k}_{\mathrm{p} 0}$ and $\mathrm{k}_{\mathrm{t}, \mathrm{b} 0}$ correspond to the propagation and bimolecular termination intrinsic rate constants respectively, i.e. without any diffusional control. $A_{p}$ and $A_{t, b}$ are the parameters which govern the rate at which, respectively, $k_{p}$ and $k_{t, b}$ decrease with viscosity. Finally, $v_{f, c p}$ and $v_{f, c t, b}$ represent the critical fractional free volumes at which propagation and bimolecular termination become diffusionlimited respectively.

$\mathrm{k}_{\mathrm{p}}=\frac{\mathrm{k}_{\mathrm{p} 0}}{\left(1+\exp \left(\mathrm{A}_{\mathrm{p}}\left(\frac{1}{v_{\mathrm{f}}}-\frac{1}{v_{\mathrm{f}, \mathrm{cp}}}\right)\right)\right)}$

$\mathrm{k}_{\mathrm{t}, \mathrm{b}}=\mathrm{k}_{\mathrm{t}, \mathrm{b} 0}\left(1+\frac{1}{\frac{\mathrm{R}_{\mathrm{rd}} \mathrm{k}_{\mathrm{p}}[\mathrm{C}=\mathrm{C}]}{\mathrm{k}_{\mathrm{t}, \mathrm{b} 0}}+\exp \left(-\mathrm{A}_{\mathrm{t}, \mathrm{b}}\left(\frac{1}{v_{\mathrm{f}}}-\frac{1}{\mathrm{v}_{\mathrm{f}, \mathrm{ct}, \mathrm{b}}}\right)\right)}\right)^{-1}$

The initiation rate constant $k_{i}$ and the PRT rate constant $k_{t, P R T}$ have the same expression as the propagation rate $k_{p}$ (Eq. 7). The exponential factors $A$ and the critical fractional free volume $v_{f, c}$ coefficients for initiation and PRT are taken equal to $A_{p}$ and $v_{f, c p}$, meaning that $k_{i}$ and $k_{t, P R T}$ start decreasing at the same moment and at the same rate as $k_{p}$ does [14]. The values of the initiation $\left(\mathrm{k}_{\mathrm{i} 0}\right)$ and PRT $\left(\mathrm{k}_{\mathrm{t}, \mathrm{PRTO}}\right)$ intrinsic rate constants depend on the nature of the primary initiating radical. As introduced in a previous paper [84], the oxygen inhibition and primary radicals (re)combination rate constants can be assimilated to the diffusion rate constant $k_{\text {diff }}$ given by the Stokes-Einstein equation (Eq. 9), as it involves the mass transport of oxygen molecules or primary radicals. Following a proposition by Buback [43], the viscosity $\eta$ exponentially increases with conversion from its initial value $\eta_{0}$, with an exponential factor $B_{\eta}$ optimized to 2 (Eq. 10) [84].

$\mathrm{k}_{\mathrm{diff}}=\frac{8 \mathrm{RT}}{3 \eta}$

$\eta=\eta_{0} \exp \left(B_{\eta} \times\right.$ Conv $)$ 


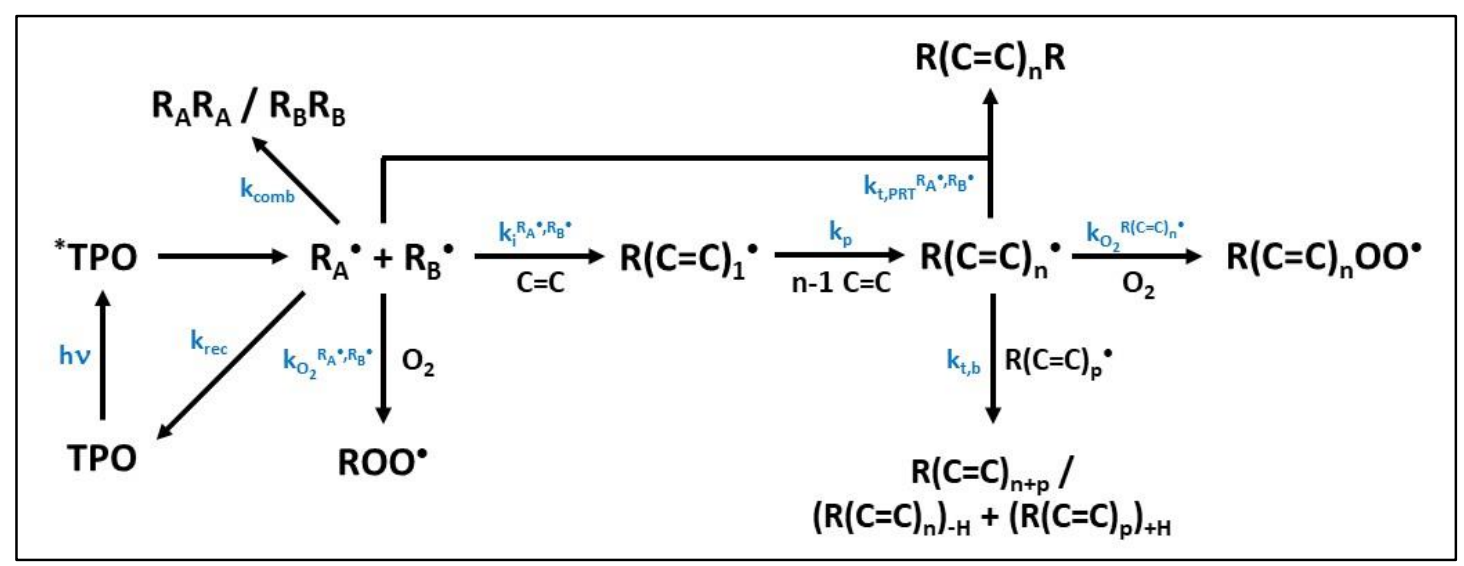

Figure 1: General mechanism of FRP initiated by TPO.

The global FRP mechanism involving TPO as a type-I PIS is given in Figure 1. Under light exposure in the UV, TPO dissociates to yield a phosphonyl $\left(R_{A}{ }^{\circ}\right)$ and a benzoyl $\left(R_{B}{ }^{*}\right)$ radicals (see Figure $\mathrm{S} 2$ in the $\mathrm{ESI}$ ). These initiating radicals then react onto a monomer $\mathrm{C}=\mathrm{C}$ double bond to initiate the growth of macroradicals $\left(\mathrm{k}_{\mathrm{i}}{ }^{\mathrm{R}^{*} \cdot \mathrm{R}^{*}}\right)$, however with a different initiating ability (intrinsic initiation rate constant $k_{i o}$ of $1.8-3.5 \times 10^{7}$ [86-93] and $1.8-3.6 \times 10^{5} \mathrm{M}^{-1} \cdot \mathrm{s}^{-1}[90,94]$ respectively). They can also combine with their equivalents to create a dimer $R_{A} R_{A}$ or $R_{B} R_{B}$ $\left(\mathrm{k}_{\mathrm{comb}}\right)$, recombine to yield TPO again $\left(\mathrm{k}_{\mathrm{rec}}\right)$, or react with a macroradical (PRT, vide infra). Macroradicals $\mathrm{R}(\mathrm{C}=\mathrm{C})_{\mathrm{n}}{ }^{\cdot}$ (whatever their chain length $\mathrm{n}$ ) grow by propagation $\left(k_{p}\right)$ or terminate either by bimolecular termination $\left(k_{t, b}\right)$ or by PRT with $R_{A}{ }^{*}$ or $R_{B}{ }^{*}\left(k_{t, P R T} R^{A^{*}, R_{B}{ }^{*}}\right)$. No distinction is done in the model between the macroradicals which propagate or are trapped in the tridimensional matrix by occlusion. In order to limit the effect of $\mathrm{O}_{2}$ inhibition on the photopolymerization kinetics, and especially on the termination reactions, experiments and modeling were performed for laminated samples, so continuous oxygen diffusion from the atmosphere is excluded. However, the initially dissolved oxygen can inhibit the photopolymerization until its concentration is sufficiently low, and the corresponding reactions have to be taken into account in the kinetic model to reproduce the inhibition time. Due to the short lifetime of TPO triplet state and its high dissociation quantum yield [95], oxygen inhibition

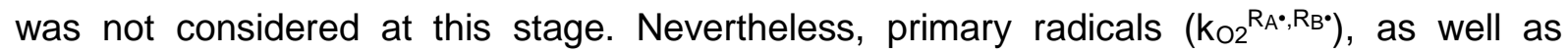
macroradicals $\left(\mathrm{KO}_{2}{ }^{\mathrm{R}(\mathrm{C}=\mathrm{C}) n^{*}}\right)$ can react with $\mathrm{O}_{2}$ to yield inactive peroxy radicals $\mathrm{ROO}^{*}$ or $\mathrm{R}(\mathrm{C}=\mathrm{C})_{n} \mathrm{OO}$. The set of differential equations corresponding to the full FRP mechanism displayed in Figure 1 is given in the ESI.

The determination of the kinetic parameters for TPO-initiated photopolymerization has been fully described in [84] and their values are listed in the ESI. Among them, the initial dissolved oxygen concentration has been optimized to $3.2 \times 10^{-4} \mathrm{M}$ by a trial-and-error fit to experimental data, in agreement with the experimental value obtained in [96] for a pure acrylic monomer. 


\subsection{Comparison to experiment}

Characteristic values for experimental FRP kinetic curves are the final conversion $\operatorname{Conv}^{\mathrm{f}}(\%)$ and the maximal rate of polymerization $\mathrm{R}_{\mathrm{p}}{ }^{\max }$. Calculated $\operatorname{Conv}(\%)$ and $\mathrm{R}_{\mathrm{p}} v s$. irradiation time curves are compared with experimental data obtained for 0.5 and $1 \mathrm{wt} \%$ of TPO under $395 \mathrm{~nm}$ LED irradiation (10 mW.cm ${ }^{-2}$ ). A good agreement between experimental and calculated curves can be seen in Figure $2 \mathrm{a}$ ) and b), respectively on the final conversion and maximal rate of polymerization values. The corresponding results are listed in Table 1. Figure 3 plots the FTIR spectra before and after light irradiation for 1 wt\% of TPO (see Figure S3 in the ESI for 0.5 wt\%).

Table 1: Comparison between experimental and simulated data for final conversion Conv ${ }^{\dagger}$ (\%) and maximal rate of polymerization $\mathrm{R}_{\mathrm{p}}{ }^{\mathrm{max}}$.

\begin{tabular}{ccccc}
\hline TPO & \multicolumn{2}{c}{$\mathbf{0 . 5} \mathbf{w t} \%$} & \multicolumn{2}{c}{$\mathbf{1}$ wt\% } \\
\hline & Experiment & Model & Experiment & Model \\
\hline Conv $^{\mathbf{f}}(\%)$ & 80 & 82.5 & 84.5 & 83.8 \\
$\mathbf{R}_{\mathbf{p}}{ }^{\text {max }}\left(\mathbf{M . s}^{-1}\right)$ & 2.7 & 2.5 & 3.3 & 3.6 \\
\hline
\end{tabular}


a)

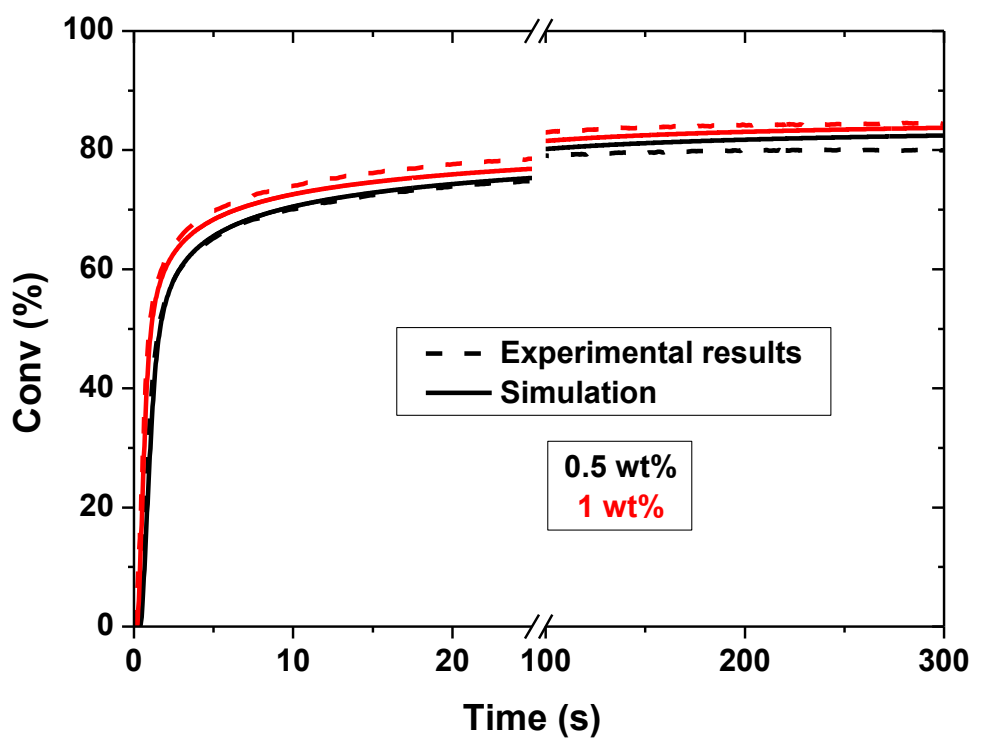

b)

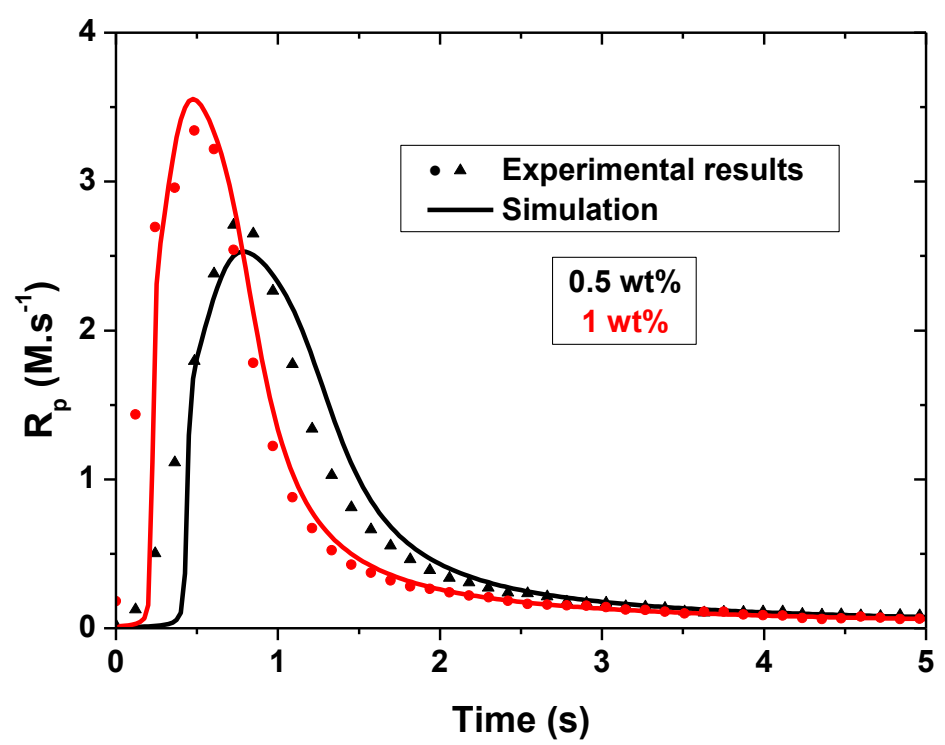

Figure 2: Comparison between experimental (dashed) and simulated (plain) a) acrylate double bond conversion b) rate of polymerization for 0.5 (black) and 1 wt $\%$ (red) of TPO $\left(\lambda_{\text {irr }}\right.$ $=395 \mathrm{~nm}, 10 \mathrm{~mW} \cdot \mathrm{cm}^{-2}$ ). 


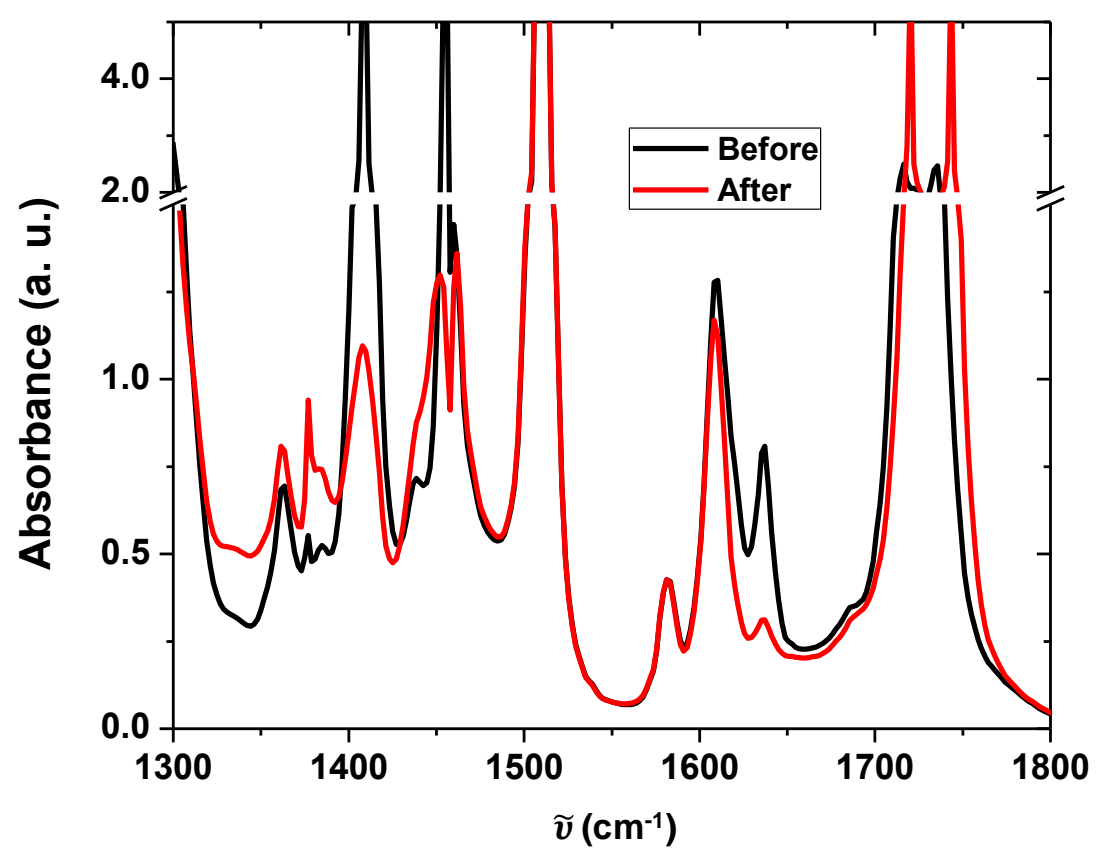

Figure 3: FTIR spectra before (black) and after (red) light irradiation (tirr $=300 \mathrm{~s}$ ) for $1 \mathrm{wt} \%$ of TPO $\left(\lambda_{\text {irr }}=395 \mathrm{~nm}, 10 \mathrm{~mW} \cdot \mathrm{cm}^{-2}\right)$.

\subsection{Oxygen inhibition in laminated samples}

As stated before, experiments and simulations were performed in the case of laminated samples. However, oxygen inhibition actually occurs until the $\mathrm{O}_{2}$ molecules initially dissolved in the resin are totally consumed. The production of inactive peroxy radicals from the reaction of oxygen on primary radicals $\left(R_{A}{ }^{*}\right.$ and $\left.R_{B}{ }^{\circ}\right)$ and macroradicals is described by the following set of differential equations:

$\frac{\mathrm{d}\left[\mathrm{R}_{\mathrm{A}} \mathrm{OO} \cdot\right]}{\mathrm{dt}}=\mathrm{k}_{\mathrm{O}_{2}} \mathrm{R}_{\mathrm{A}}^{\cdot}\left[\mathrm{R}_{\mathrm{A}}^{\cdot}\right]\left[\mathrm{O}_{2}\right]$

$\frac{\mathrm{d}\left[\mathrm{R}_{\mathrm{B}} \mathrm{O0^{* }}\right]}{\mathrm{dt}}=\mathrm{k}_{\mathrm{O}_{2}} \mathrm{R}_{\mathrm{B}}^{\cdot}\left[\mathrm{R}_{\mathrm{B}}^{\cdot}\right]\left[\mathrm{O}_{2}\right]$

$\frac{\mathrm{d}\left[\mathrm{R}(\mathrm{C}=\mathrm{C})_{\mathrm{n}} \mathrm{O0^{* }}\right]}{\mathrm{dt}}=\mathrm{k}_{\mathrm{O}_{2}}{ }^{\mathrm{R}(\mathrm{C}=\mathrm{C})_{\mathrm{n}}} \cdot\left[\mathrm{R}(\mathrm{C}=\mathrm{C})_{\mathrm{n}}{ }^{\bullet}\right]\left[\mathrm{O}_{2}\right]$

$\mathrm{R}_{\mathrm{A}} \mathrm{OO}$ (Eq. 11) and $\mathrm{R}_{\mathrm{B}} \mathrm{OO}$ (Eq. 12) are the inhibited primary initiating radicals and $\mathrm{R}(\mathrm{C}=\mathrm{C})_{n} \mathrm{OO}{ }^{*}$ (Eq. 13) the inhibited macroradicals. The temporal evolution of $\left[\mathrm{R}_{\mathrm{A}} \mathrm{OO} \mathrm{O}^{*}\right],\left[\mathrm{R}_{\mathrm{B}} \mathrm{OO}^{\circ}\right]$, and $\left[\mathrm{R}(\mathrm{C}=\mathrm{C})_{n} \mathrm{OO} \mathrm{O}^{\circ}\right.$ (on a logarithmic scale) is compared to that of oxygen for $0.5 \mathrm{wt} \%$ TPO in Figure 4 (for 1 wt\%, see Figure S4 in the ESI). As known, the initially dissolved oxygen is rapidly consumed ( 0.45 and $0.22 \mathrm{~s}$ for 0.5 and $1 \mathrm{wt} \%$ TPO, respectively). This consumption is faster for the most concentrated formulation by means of a faster generation of 
(macro)radicals. More interestingly, it is also shown that the concentration of oxygen-inhibited macroradicals $\left[\mathrm{R}(\mathrm{C}=\mathrm{C})_{\mathrm{n}} \mathrm{OO^{* }}\right]$ is orders of magnitude higher than that of primary peroxy radicals $\left[\mathrm{R}_{\mathrm{A}} \mathrm{OO}^{\circ}\right]$ and $\left[\mathrm{R}_{\mathrm{B}} \mathrm{OO} \mathrm{O}^{\circ}\right.$.

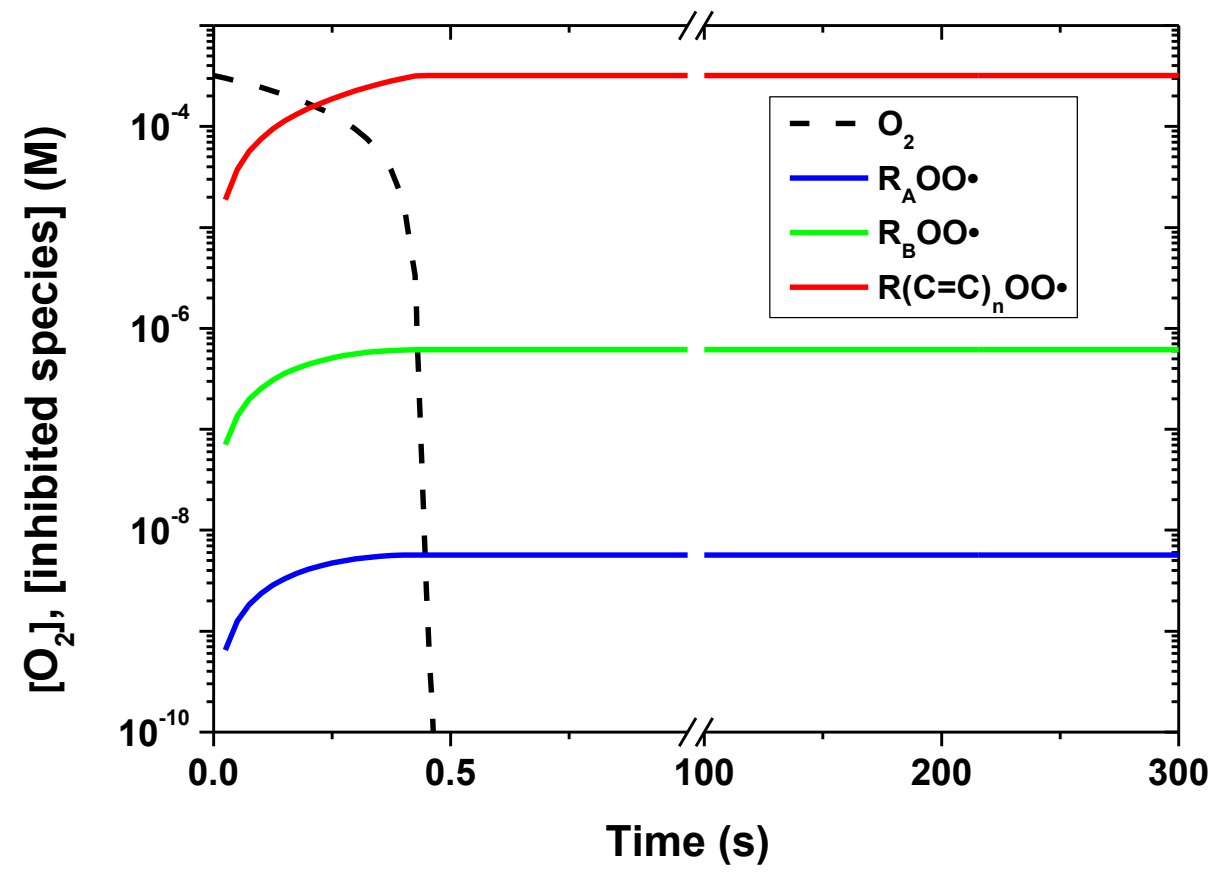

Figure 4: Evolution of the oxygen and inactive peroxy radical (primary and macroradicals) concentrations (logarithmic scale) as a function of the irradiation time $\left(0.5 \mathrm{wt} \% \mathrm{TPO}, \lambda_{\text {irr }}=\right.$ $\left.395 \mathrm{~nm}, 10 \mathrm{~mW} . \mathrm{cm}^{-2}\right)$.

This thus reveals that the main species reacting with the initially dissolved oxygen are the macroradicals $\mathrm{R}(\mathrm{C}=\mathrm{C})_{\mathrm{n}}{ }^{\circ}$, and that, contrary to what is generally stated, the initiating radicals almost do not contribute to the oxygen consumption. Indeed, more than $99.8 \%$ of the initial oxygen is consumed through this reaction. The quasi-absence of inhibition on the initiating primary radicals $R_{A}{ }^{\cdot}$ and $R_{B}{ }^{\cdot}$ can be explained by their relative reactivities towards acrylic double bonds and oxygen. Indeed, with almost equivalent rate constants for initiation and oxygen inhibition, primary radicals preferentially react with highly concentrated monomer $\mathrm{C}=\mathrm{C}$ double bonds $\left([\mathrm{C}=\mathrm{C}]_{0} \sim 4.5 \mathrm{M}\right)$ than with low-concentrated oxygen $\left(\left[\mathrm{O}_{2}\right]_{0} \sim 3.2 \times 10^{-4} \mathrm{M}\right)$. The kinetic competition between propagation and inhibition is more balanced, explaining why the macroradicals are the main $\mathrm{O}_{2}$ trapping agents during the inhibition process. A more detailed discussion of the competition between oxygen inhibition and the other reactions of the process (initiation, propagation and termination) is proposed in the ESI. 


\subsection{Termination reactions}

Three different bimolecular termination reactions can occur during FRP: $i$. formation of a chemical bond between two macroradicals (combination), ii. hydrogen abstraction from a macroradical to a second one with formation of a double bond on the former (disproportion), iii. reaction between a primary radical and a macroradical (PRT). As they both involve a reaction between two macroradicals, combination and disproportion are lumped into a single mechanism called bimolecular termination $\left(k_{t, b}\right)$, while PRT by $R_{A}{ }^{*}$ and $R_{B}{ }^{*}$ are distinguished $\left(k_{t, P R T} R^{A^{*}}\right.$ and $\left.k_{t, P R T} R^{R^{*}}\right)$. It is possible to model these termination reactions by the following set of differential equations:

$\frac{\mathrm{d}[\mathrm{bimol}]}{\mathrm{dt}}=2 \mathrm{k}_{\mathrm{t}, \mathrm{b}}\left[\mathrm{R}(\mathrm{C}=\mathrm{C})_{\mathrm{n}}{ }^{\bullet}\right]^{2}$

$\frac{\mathrm{d}\left[\mathrm{R}(\mathrm{C}=\mathrm{C})_{\mathrm{n}} \mathrm{R}_{\mathrm{A}}\right]}{\mathrm{dt}}=\mathrm{k}_{\mathrm{t}, \mathrm{PRT}} \mathrm{R}^{\cdot}\left[\mathrm{R}(\mathrm{C}=\mathrm{C})_{\mathrm{n}}{ }^{\cdot}\right]\left[\mathrm{R}_{\mathrm{A}}{ }^{\cdot}\right]$

$\frac{\mathrm{d}\left[\mathrm{R}(\mathrm{C}=\mathrm{C})_{\mathrm{n}} \mathrm{R}_{\mathrm{B}}\right]}{\mathrm{dt}}=\mathrm{k}_{\mathrm{t}, \mathrm{PRT}} \mathrm{R}^{\mathrm{R}^{\circ}}\left[\mathrm{R}(\mathrm{C}=\mathrm{C})_{\mathrm{n}}{ }^{\cdot}\right]\left[\mathrm{R}_{\mathrm{B}}{ }^{\cdot}\right]$

[bimol] represents the concentration of macroradicals terminated by bimolecular termination (combination or disproportion), either diffusional or through reaction diffusion (Eq. 14). $\left[R(C=C)_{n} R_{A}\right]$ and $\left[R(C=C)_{n} R_{B}\right]$ stand for the concentrations of macroradicals terminated through PRT by $R_{A}{ }^{*}$ and $R_{B} \cdot$ respectively (Eqs. 15 and 16). At final conversion, the polymer network is vitrified and it can be assumed that all remaining macroradicals are trapped by occlusion in the tridimensional network.

Evolution of [bimol], $\left[R(C=C)_{n} R_{A}\right],\left[R(C=C)_{n} R_{B}\right]$ and $\left[R(C=C)_{n}{ }^{\circ}\right]$ as a function of irradiation time is plotted in Figure 5 for 1 wt \% TPO (see Figure S5 in the ESI for 0.5 wt\%). It can be seen that the concentration of macroradicals terminated by bimolecular reactions increases from the onset to the end of the polymerization. It is largely higher than $\left[R(C=C)_{n} R_{A}\right]$ and $\left[R(C=C)_{n} R_{B}\right]$ which also continuously increase, or $\left[R(C=C)_{n}{ }^{*}\right]$ which increases up to $100 \mathrm{~s}$ and finally decreases until the polymerization ceases. Moreover, the growth of $\left[R(C=C)_{n} R_{A}\right]$ and $\left[R(C=C)_{n} R_{B}\right]$ starts at longer times than that of macroradicals terminated by bimolecular termination. Considering the final values of these concentrations, the fractions of macroradicals terminated by each termination mode at the end of the photopolymerization can be calculated and the corresponding results are given in Table 2. 


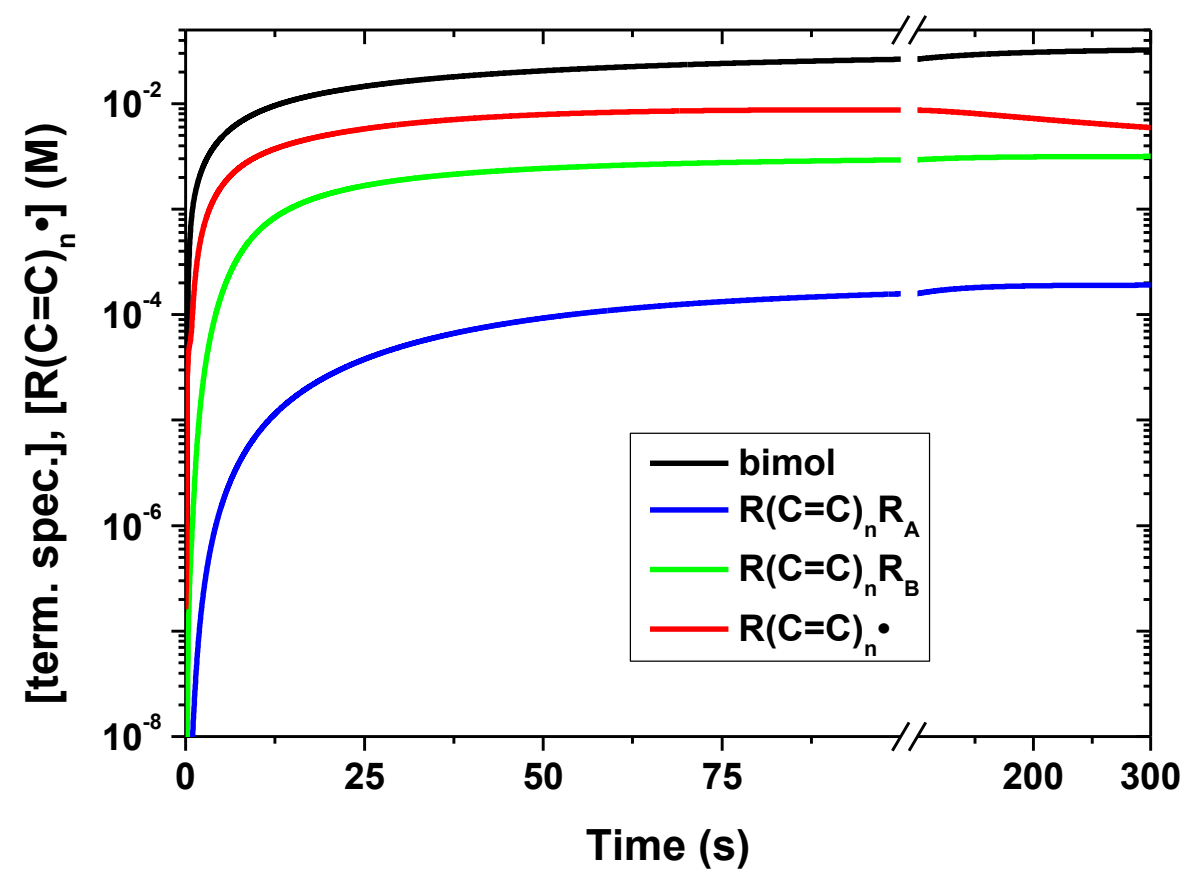

Figure 5: Evolution of the terminated species and macroradical concentrations (logarithmic scale) as a function of the irradiation time ( $\left.1 \mathrm{wt} \% \mathrm{TPO}, \lambda_{\text {irr }}=395 \mathrm{~nm}, 10 \mathrm{~mW} . \mathrm{cm}^{-2}\right)$.

Table 2: Relative ratio of macroradical termination reactions at final conversion ( $t_{\text {irr }}=300 \mathrm{~s}$ ).

\begin{tabular}{ccccc}
\hline \multicolumn{5}{c}{ Termination mode (\%) } \\
\hline TPO & Bimolecular & PRT by $\mathbf{R}_{\mathbf{A}}{ }^{*}$ & ${\text { PRT by } \mathbf{R}_{\mathbf{B}}}^{*}$ & Occlusion $^{*}$ \\
\hline 0.5 wt\% & 79.9 & 0.2 & 6.8 & 13.1 \\
1 wt\% & 77.7 & 0.4 & 7.6 & 14.3 \\
\hline
\end{tabular}

From Figure 5 and Table 2, it can be seen that bimolecular termination is actually the main termination mode at the end of the reaction, followed by occlusion and PRT by $R_{B}{ }^{\circ}$. In comparison, PRT by $R_{A} \cdot$ is only a minor way of termination. Relative values of $\left[R(C=C)_{n} R_{A}\right]$ and $\left[R(C=C)_{n} R_{B}\right]$ also show that $P R T$ by benzoyl $R_{B} \cdot$ radicals is more efficient than that by phosphonyl $R_{A}{ }^{*}$ ones, a fact which can be explained by their different initiation efficiencies. Indeed, intrinsic initiation rate constant $k_{i 0}$ is 100 times higher for the phosphonyl radical $R_{A}$. (1.8-3.5 $\left.\times 10^{7}[86-93]\right)$ than for its benzoyl equivalent $R_{B} \cdot\left(1.8-3.6 \times 10^{5} \mathrm{M}^{-1} \cdot \mathrm{s}^{-1}[90,94]\right)$. The latter thus becomes more available for PRT reactions.

A deeper insight into the evolution of termination modes all along the photopolymerization is given in Figure 6, where the fractions of macroradicals and terminated species are 
represented as a function of the acrylate conversion (for $1 \mathrm{wt} \%$ TPO) all along the polymerization. The corresponding change in TPO concentration is also plotted for discussion.

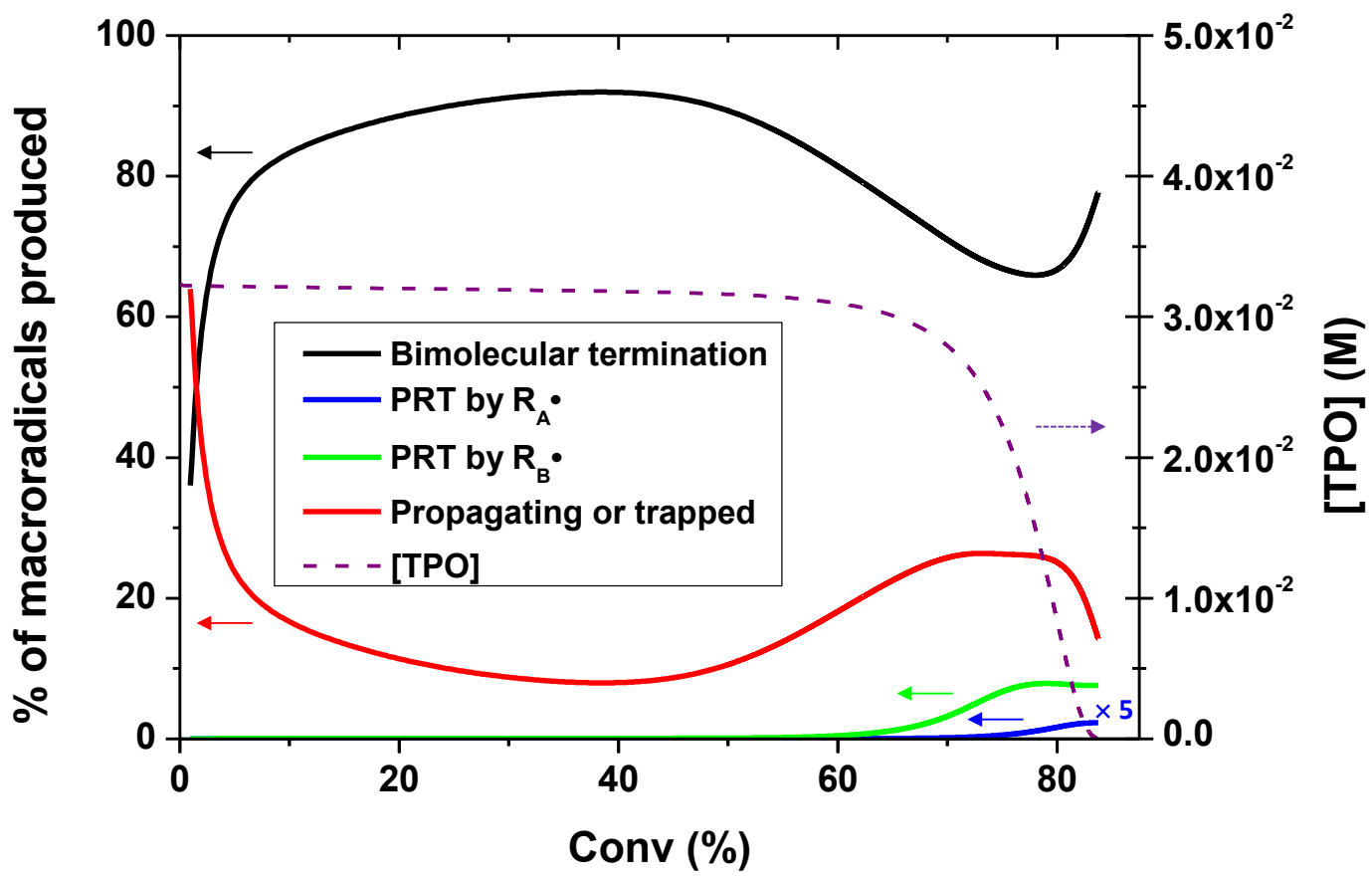

Figure 6: Evolution of the fractions of terminated species and propagating or trapped macroradicals (left) and of the TPO concentration (right) as a function of the acrylate conversion ( $1 \mathrm{wt} \%$ TPO, $\lambda_{\text {irr }}=395 \mathrm{~nm}, 10 \mathrm{~mW} . \mathrm{cm}^{-2}$ ). The horizontal arrows indicate the corresponding vertical axis - blue curve is magnified by a factor 5 for clarity.

It can be seen that the ratio of these different pathways actually evolves throughout the polymerization process. Indeed, the bimolecular fraction increases up to $92 \%$ at $40 \%$ of conversion, then decreases before a final increase at around $80 \%$ of conversion. In parallel, the fraction of macroradicals decreases from the start of the polymerization process until $40 \%$ of conversion, increases up to a plateau value around $70 \%$ of conversion and finally decreases after $80 \%$ of conversion. Termination by $\mathrm{R}_{\mathrm{B}} \cdot$ radicals is only significant for a conversion higher than $50 \%$. It first increases, before a slight decrease at the end of the process. The noticeable role of $R_{A} \cdot$ radicals in termination is lower and delayed to higher conversions (> $70 \%$ ), with a similar evolution than that of $\mathrm{R}_{\mathrm{B}}$. Finally, Figure 6 also shows that TPO photolysis occurs at high conversion (when the process is slowed down), its concentration being almost constant up to $60 \%$ of conversion.

Bimolecular termination is clearly the main termination process throughout the polymerization, even at high conversion. This result is consistent with the introduction of 10 
wt\% of DMSO in the model resin used here, these small molecules playing the role of plasticizers at high conversion. The strong growth of the bimolecular termination during the first $20 \%$ of conversion can be explained by the continuous production of macroradicals as initiation occurs, associated to the high value of $\mathrm{k}_{\mathrm{t}, \mathrm{b}}$ in this range (represented on a logarithmic scale in Figure 7). Despite its decrease (due to the diffusion control of pure bimolecular termination), the effective $\mathrm{k}_{\mathrm{t}, \mathrm{b}}$ stays higher than $2 \times 10^{5} \mathrm{M}^{-1} \cdot \mathrm{s}^{-1}$ and thus overcomes the other termination modes. As PRT is not yet significant, the fraction of macroradicals is consequently reduced. $\mathrm{k}_{\mathrm{t}, \mathrm{b}}$ stays almost constant from 20 to $40 \%$ of conversion because termination by reaction diffusion takes over. Indeed, $\mathrm{k}_{\mathrm{t}, \mathrm{b}}$ becomes then proportional to $\mathrm{k}_{\mathrm{p}}$ (see Eq. 1). As can be seen in Figure $7, k_{p}$ stays constant up to $40 \%$ of conversion because propagation becomes diffusion-controlled at a higher conversion than bimolecular termination (small monomer molecules are more free to diffuse than macroradicals). As a consequence, the increase of bimolecular termination (mainly by reaction diffusion) carries on between 20 and $40 \%$ of conversion but is slower (Figure 6), and the fraction of macroradicals decreases (PRT is still not yet efficient).

The decrease of bimolecular termination after $40 \%$ of conversion is also associated to the proportionality between $\mathrm{k}_{\mathrm{t}, \mathrm{b}}$ and $\mathrm{k}_{\mathrm{p}}$. Indeed, propagation becomes diffusion-controlled at high conversion (here after $40 \%$ of conversion) and effective $k_{p}$ falls (see Figure 7 ). The reaction diffusion rate constant being proportional to $\mathrm{k}_{\mathrm{p}}$ (Eq. 1), $\mathrm{k}_{\mathrm{t}, \mathrm{b}}$ also diminishes and bimolecular termination then decreases. As PRT is also not significant in this range, the amount of macroradicals consequently raises. The predominance of bimolecular termination over PRT throughout the polymerization can be explained by its higher rate compared to that of PRT (see Figure S6 in the ESI). The evolution of $k_{p}$ and $k_{t, b}$ all along the photopolymerization is actually in agreement with what have been experimentally observed [43,48,49,79] and simulated $[43,46,79]$. 


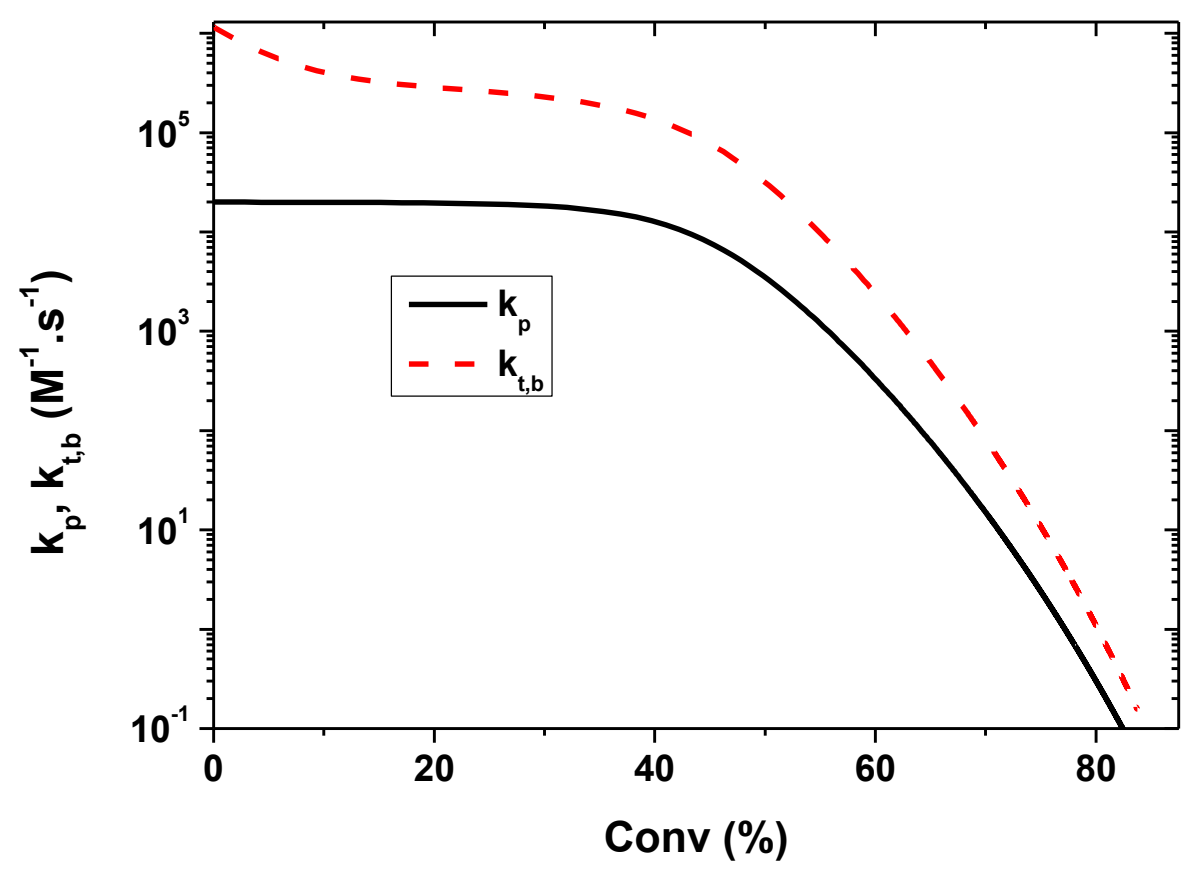

Figure 7: Effective propagation ( $\mathrm{k}_{\mathrm{p}}$, black) and bimolecular termination $\left(\mathrm{k}_{\mathrm{t}, \mathrm{b}}\right.$, red) rate constants (logarithmic scale) as a function of the acrylate conversion ( $1 \mathrm{wt} \% \mathrm{TPO}, \lambda_{\text {irr }}=395$ $\left.\mathrm{nm}, 10 \mathrm{~mW} . \mathrm{cm}^{-2}\right)$.

As previously mentioned, PRT becomes efficient only in the last stages of the process. This delayed effectiveness of PRT has to be related to the competition between PRT and initiation reactions for the primary radicals. This can be expressed by the ratio between the PRT rate $R_{t, P R T}=k_{t, P R T}\left[R(C=C)_{n}{ }^{*}\right]\left[R^{*}\right]$ and the initiation rate $R_{i}=k_{i}[C=C]\left[R^{*}\right]$ (with $R^{*}=R_{A}{ }^{*}$ or $R_{B}{ }^{*}$, Eq. 17). The evolution of this ratio as a function of polymer conversion is given in Figure 8.

$\mathrm{R}_{\mathrm{t}, \mathrm{PRT}} / \mathrm{R}_{\mathrm{i}}=\frac{\mathrm{k}_{\mathrm{t}, \mathrm{PRT}}\left[\mathrm{R}(\mathrm{C}=\mathrm{C})_{\mathrm{n}}{ }^{\bullet}\right]\left[\mathrm{R}^{\bullet}\right]}{\mathrm{k}_{\mathrm{i}}[\mathrm{C}=\mathrm{C}]\left[\mathrm{R}^{\bullet}\right]}$ 


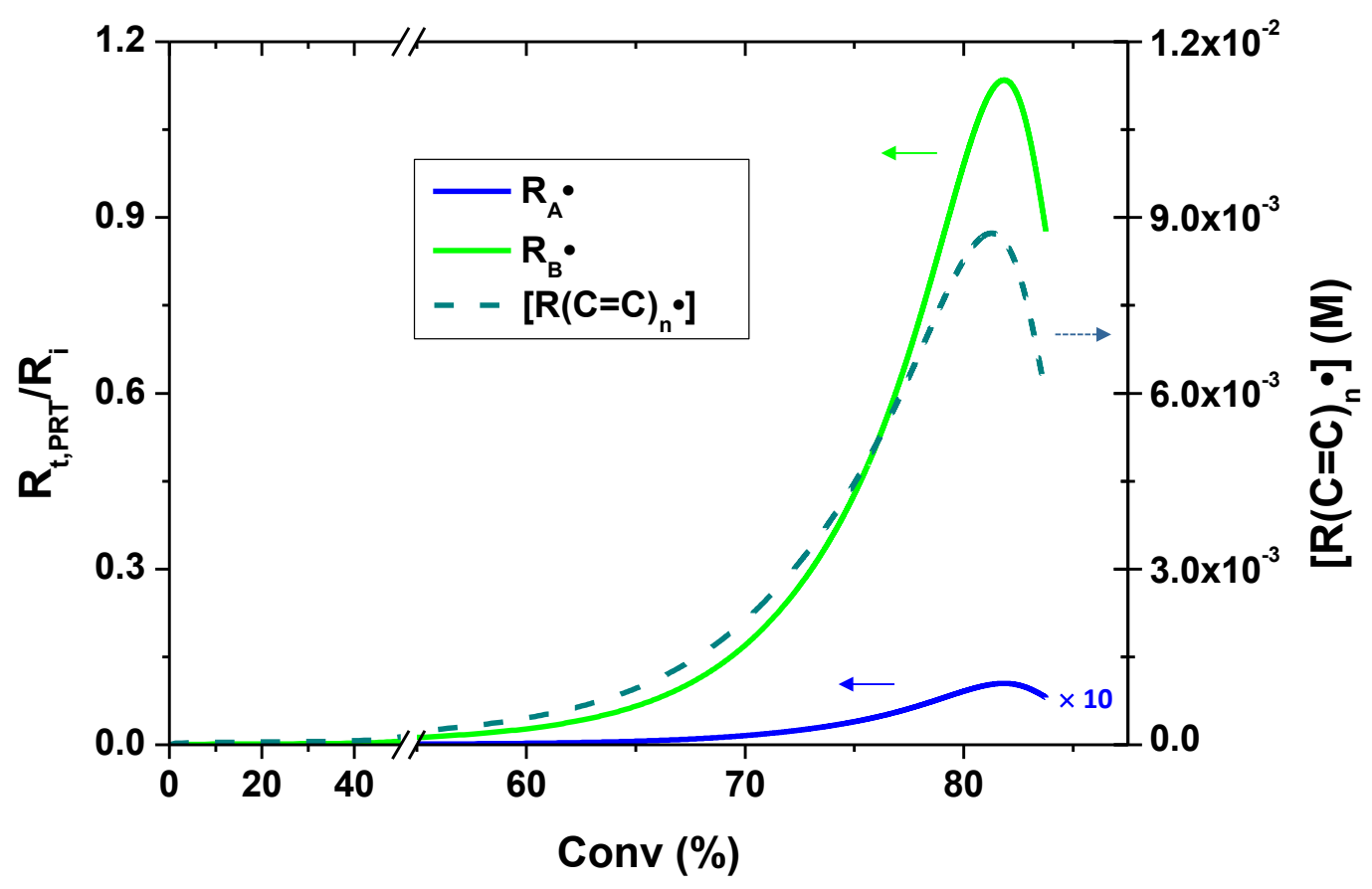

Figure 8: Evolution of the $R_{t, P R T} / R_{i}$ ratio for $R_{A}{ }^{\cdot}$ (green) and $R_{B}{ }^{*}$ (blue) radicals (left) and of $\left[R(C=C)_{n}{ }^{*}\right]$ (dashed, right) as a function of the acrylate conversion ( $1 \mathrm{wt} \% \mathrm{TPO}, \lambda_{\mathrm{irr}}=395 \mathrm{~nm}$, $\left.10 \mathrm{~mW} . \mathrm{cm}^{-2}\right)$. The horizontal arrows indicate the corresponding vertical axis - blue curve is magnified by a factor 10 for clarity.

It can be seen that the $R_{t, P R T} / R_{i}$ ratio is close to zero for a large part of the process, only increasing after $50 \%$ of conversion for both $R_{B}{ }^{\cdot}$ and $R_{A}{ }^{*}$. After a maximum at $82 \%$ of conversion, the ratio finally falls for both primary radicals. However, it has to be noticed that the ratio for phosphonyl $R_{A} \cdot$ radicals is largely lower than that for benzoyl $R_{B}{ }^{\cdot}$ ones throughout the process. An initial low value of $R_{t, P R T} / R_{i}$ means that initiation is largely more efficient than PRT for a large part of the FRP reaction. The following raise shows that competition of PRT with initiation becomes more efficient when the conversion increases. Indeed, the effective $\mathrm{k}_{\mathrm{t}, \mathrm{PRT}}$ and $\mathrm{k}_{\mathrm{i}}$ rate constants decrease by means of the progressive diffusional control of the associated reactions during the process. In the model, the exponential factor $A$ and critical free volume $v_{f, c}$ parameters are identical for both $k_{t, P R T}$ and $k_{i}$ (equal to $A_{p}$ and $v_{f, c p}$, vide supra), thus the reaction rate constants start decreasing at the same time and at the same rate. In parallel, the monomer concentration $[\mathrm{C}=\mathrm{C}]$ also decreases all along the polymerization while $\left[\mathrm{R}(\mathrm{C}=\mathrm{C})_{n}{ }^{*}\right]$ increases due to the decrease of $\mathrm{k}_{\mathrm{t}, \mathrm{b}}$ (vide supra), with a maximum around $82 \%$ of conversion (Figure 8). As a consequence, the $R_{t, P R T} / R_{i}$ ratio increases as long as $\left[R(C=C)_{n}{ }^{\circ}\right]$ raises. PRT becomes hence more efficient, and its proportion in the termination modes increases (see Figure 6). The lower $R_{t, P R T} / R_{i}$ ratio for $R_{A}{ }^{*}$ could explain the delay in the significant role of PRT 
by $R_{A}{ }^{*}$ in Figure 6 . This is caused by the higher intrinsic initiation rate constant $k_{i 0}$ for phosphonyl radicals $R_{A}{ }^{\cdot}$ than for benzoyl $R_{B}{ }^{\cdot}$ ones. Initiation by $R_{A}{ }^{\cdot}$ thus longer prevails over PRT and the concentration of $R_{A}{ }^{\circ}$ stays lower than that of $R_{B}{ }^{\circ}$. This emphasizes that the primary radicals may play a dual role during the photopolymerization, either initiator of the reaction or terminating agent. The choice of the photoinitiator structure would also have to take into account this effect, in addition to the classical requirements (absorption region, dissociation efficiency...) to optimize the polymerization performances. The increase of PRT by $R_{B}{ }^{\cdot}$ after $60 \%$ of conversion seems to impact only the bimolecular termination fraction, as a plateau is observed in the fraction of macroradicals (Figure 6 ). The final decrease of $R_{t, P R T} / R_{i}$ ratio after $82 \%$ of conversion is associated to the decrease of $\left[R(C=C)_{n}{ }^{*}\right]$ (Figure 8$),\left[R_{A}{ }^{*}\right]$ and $\left[R_{B}{ }^{*}\right]$, which could be due to the almost complete photolysis of TPO and the cessation of (macro)radical formation.

Figures 6 and 8 both present a strong change at around $80 \%$ of conversion. This has to be related to the evolution of [TPO] plotted in Figure 6. Indeed, most part of the initial TPO is consumed after $80 \%$ of conversion, so the production of primary initiating radicals falls. Initiation and PRT are then strongly reduced. As a consequence of the reduction of initiation, less macroradicals are produced (up to a complete cessation when TPO totally disappears). However, bimolecular termination still occurs between the remaining macroradicals, so its fraction increases while that of macroradical decreases. This final enhancement of bimolecular termination explains the final decrease of $\left[R(C=C)_{n}{ }^{*}\right]$ observed in Figure 8 , causing that of the $R_{t, P R T} / R_{i}$ ratio and thus the final decline of PRT fraction for both initiating radicals.

\subsection{Effect of experimental parameters $I_{0}^{s}$ and [TPO]o}

The incident light intensity on the surface sample $I_{0}^{s}$ and/or the initial photoinitiator concentration are generally tuned in order to optimize the photopolymerization process. However, a modification of these experimental parameters will affect the initiation and termination reactions. Kinetic modeling represents an efficient way to quantify this influence with numerical values, especially that on the termination modes.

\subsubsection{Influence of incident light intensity $I_{0}^{s}$ on termination reactions}

The ability of the kinetic model to reproduce changes in the incident light intensity was confirmed with photopolymerization experiments made at 2 and $5 \mathrm{~mW} . \mathrm{cm}^{-2}$ (see Figures $\mathrm{S} 7$ to $\mathrm{S} 10$ in the ESI). The effect of incident light intensity (from 2 to $50 \mathrm{~mW} . \mathrm{cm}^{-2}$ ) on conversion and $R_{p}$ curves is shown in Figure 9. Final conversion is almost not affected: only a slight lowering can be observed at higher $1_{0}{ }^{\mathrm{s}}$. However, it is clear that the rate of free radical photopolymerization is enhanced and the inhibition time reduced. As expected, the shape of 
$R_{p} v s$. irradiation time curves is strongly modified when $I_{0}{ }^{s}$ is raised: $i$. the inhibition time is reduced, ii. the $\mathrm{R}_{p}{ }^{\max }$ position is switched to shorter times, iii. the $\mathrm{R}_{p}{ }^{\max }$ values are greater, $i v$. the curves are sharper, revealing both faster autoacceleration and autodeceleration processes.
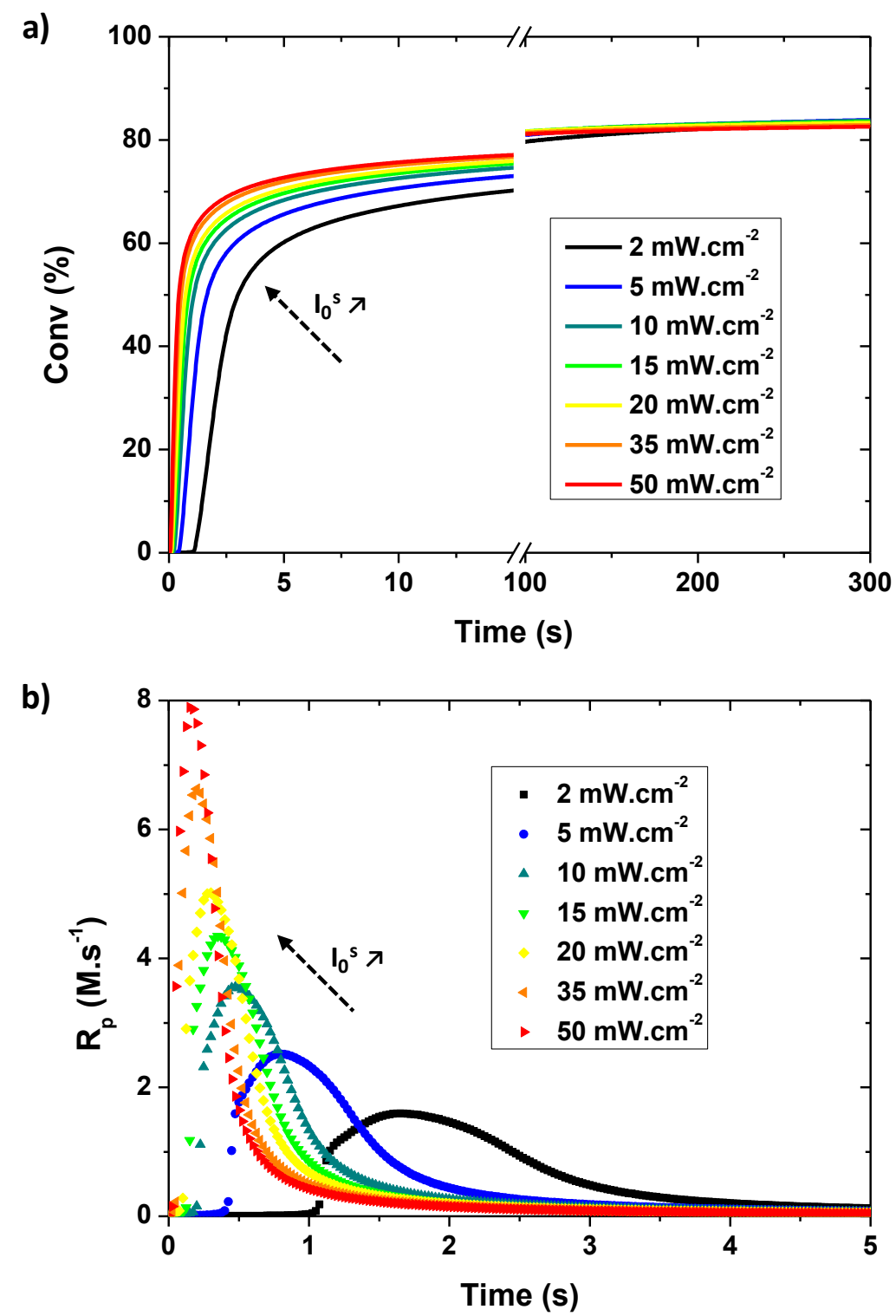

Figure 9: Effect of the incident light intensity $1_{0}^{\mathrm{s}}$ on the simulated a) conversion b) rate of polymerization ( $1 \mathrm{wt} \%$ TPO, $\lambda_{\text {irr }}=395 \mathrm{~nm}$ ).

Increasing the incident intensity enhances the rate of primary radical production at early times, hence that of macroradical formation (see Figure S11 in the ESI). As the macroradicals are the principal scavengers of dissolved $\mathrm{O}_{2}$ molecules in laminated systems (vide supra), this faster production of macroradicals explains the reduced inhibition time with increasing incident intensity. Moreover, the vitrification of the reaction medium occurs faster, consequently leading 
to an earlier and faster autoacceleration process (i.e. diffusion control of bimolecular termination). For the same reason, autodeceleration (i.e. diffusion control of propagation) occurs faster. The higher amount of photogenerated initiating radicals and macroradicals per second at higher intensities should explain the higher maximum rates of polymerization observed.

Increasing the incident intensity $\mathrm{I}_{0} \mathrm{~s}$ has a strong impact on termination proportions at final conversion, as shown in Figure 10. Indeed, both bimolecular termination and PRT fractions increase with $\mathrm{I}_{0}^{\mathrm{s}}$, while occlusion falls. They all reach a plateau at higher incident intensities. It clearly shows that, contrary to what is generally suggested [14], PRT never becomes the major way of termination, even at high light intensities. The increase of bimolecular termination and the reduction of radical trapping by occlusion are surprising. Indeed, it is generally stated in the literature that increasing incident intensity causes an increase of both PRT, by means of a higher concentration of primary radicals $[97,98]$, and occlusion at final conversion, due to a faster vitrification of the reaction medium [26-28].

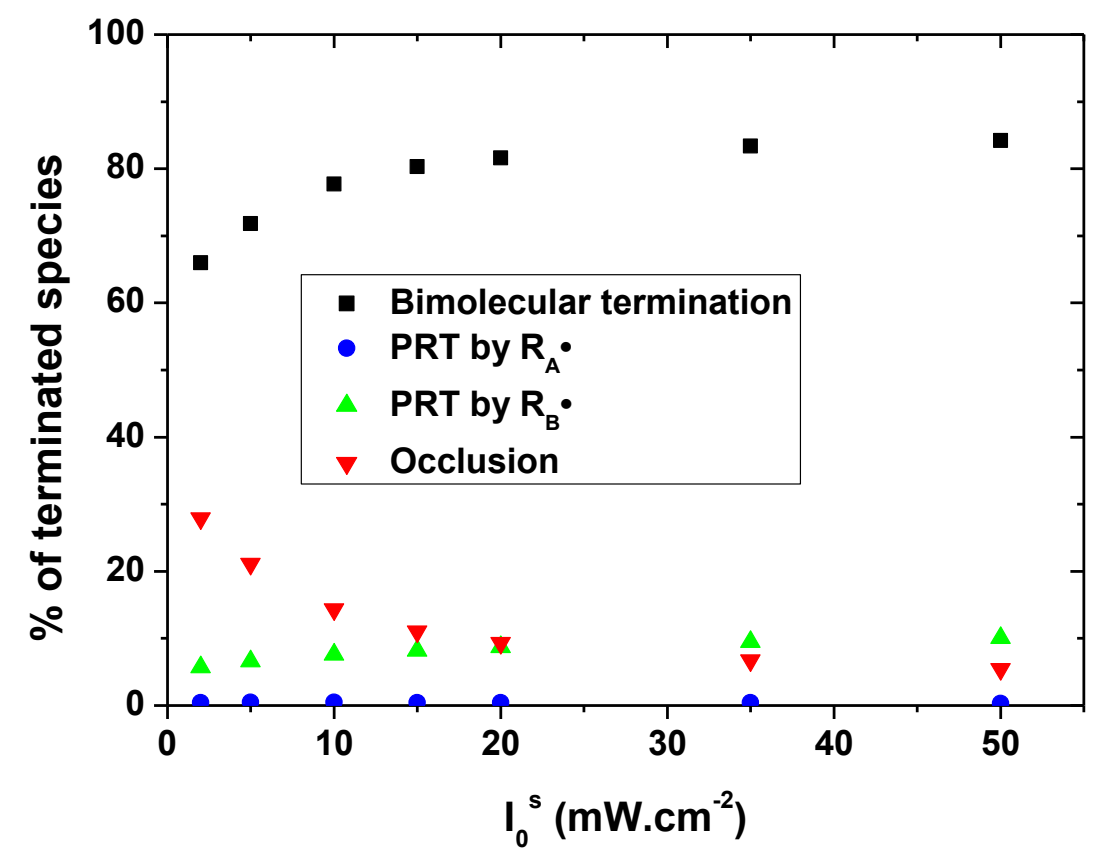

Figure 10: Influence of the incident light intensity $1_{0}^{\mathrm{s}}$ on the termination modes at final conversion ( $1 \mathrm{wt} \%$ TPO, $\lambda_{\text {irr }}=395 \mathrm{~nm}$ ).

In order to understand the influence of $10^{\mathrm{s}}$ on the final termination ratio, the change in the fractions of terminated species and macroradicals for different light intensities is compared to that of TPO concentration (Figure 11). For a given light intensity, the successive steps in the change of bimolecular termination, PRT and macroradical fractions are identical to that 
discussed in the previous section. When $\mathrm{I}_{0} \mathrm{~s}$ is modified, change in these fractions is also identical before $60 \%$ of conversion, but reveals interesting differences after. Indeed, the PRT fraction increases earlier and becomes higher when $I_{0}{ }^{s}$ is enhanced for both $R_{A}{ }^{\circ}$ and $R_{B}{ }^{*}$. It also decreases sooner at the end of the process. The final increase of bimolecular termination, as well as the corresponding decrease of macroradical fraction, is also shifted to shorter times when the incident intensity is increased. Finally, the TPO photolysis is faster when $1_{0}^{\mathrm{s}}$ is enhanced, by means of a higher amount of photons per second being absorbed by the photoinitiator molecules.

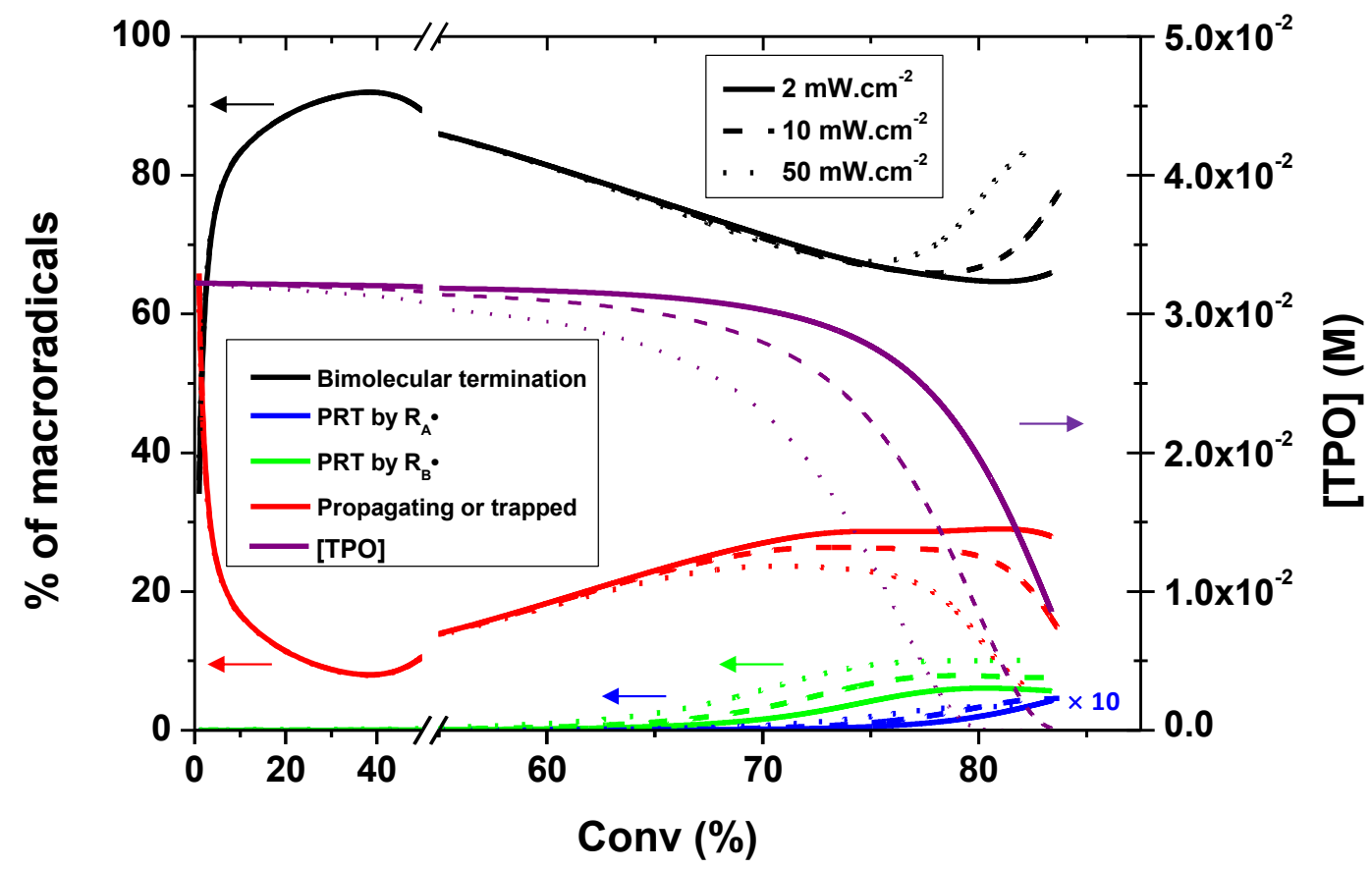

Figure 11: Change in the fractions of terminated species and macroradicals (left) and of the TPO concentration (right) for 2 (solid), 10 (dashed) and 50 (dotted) $\mathrm{mW} . \mathrm{cm}^{-2}$ as a function of the acrylate conversion ( $1 \mathrm{wt} \%$ TPO, $\lambda_{\text {irr }}=395 \mathrm{~nm}$ ). The horizontal arrows indicate the corresponding axis - blue curve is magnified by a factor 10 for clarity.

The earlier increase of PRT could be explained by a higher rate of macroradical production when $\mathrm{I}_{0}^{\mathrm{s}}$ is increased. This shifts the efficient competition between initiation and PRT reactions for primary radicals to a lower conversion, a fact supported by an earlier enhancement of the $R_{t, P R T} / R_{i}$ ratio when $I_{0}^{s}$ is increased (see Figure $S 12$ in the ESI). PRT has then more time to occur until the end of the process, and finally represents a higher amount of terminated species. The faster photolysis of TPO with increasing $\mathrm{I}_{0} \mathrm{~s}$ causes an earlier increase of bimolecular termination and a decrease of the macroradical and PRT fractions at the end of the polymerization. Indeed, as initiation ceases earlier during the process, the formation of 
macroradicals also stops sooner. Bimolecular termination has then more time to occur between the remaining macroradicals. The final fraction of bimolecular termination is then enhanced, while that of radical trapping by occlusion is consequently reduced. As TPO is consumed, PRT efficiency is also reduced because of the decrease of the primary radical formation. Its fraction then decreases earlier when $\mathrm{I}_{0}^{\mathrm{s}}$ is enhanced.

The influence of the incident light intensity on the temporal evolution of the macroradical concentration is especially interesting because the steady-state assumption is generally applied to $\left[R(C=C)_{n}{ }^{*}\right]$ in order to derive relations between the rate of polymerization $R_{p}$ and the incident light intensity $[6,8,9,25]$. This assumption notably implies that $\left[R(C=C)_{n}{ }^{*}\right]$ is constant throughout the polymerization, hence $d\left[R(C=C)_{n} \cdot\right] / d t=0$. Figure 12 shows the evolution of the rate of polymerization $R_{p}$ and that of $d\left[R(C=C)_{n}{ }^{*}\right] / d t$ as a function of the conversion for different light intensities.

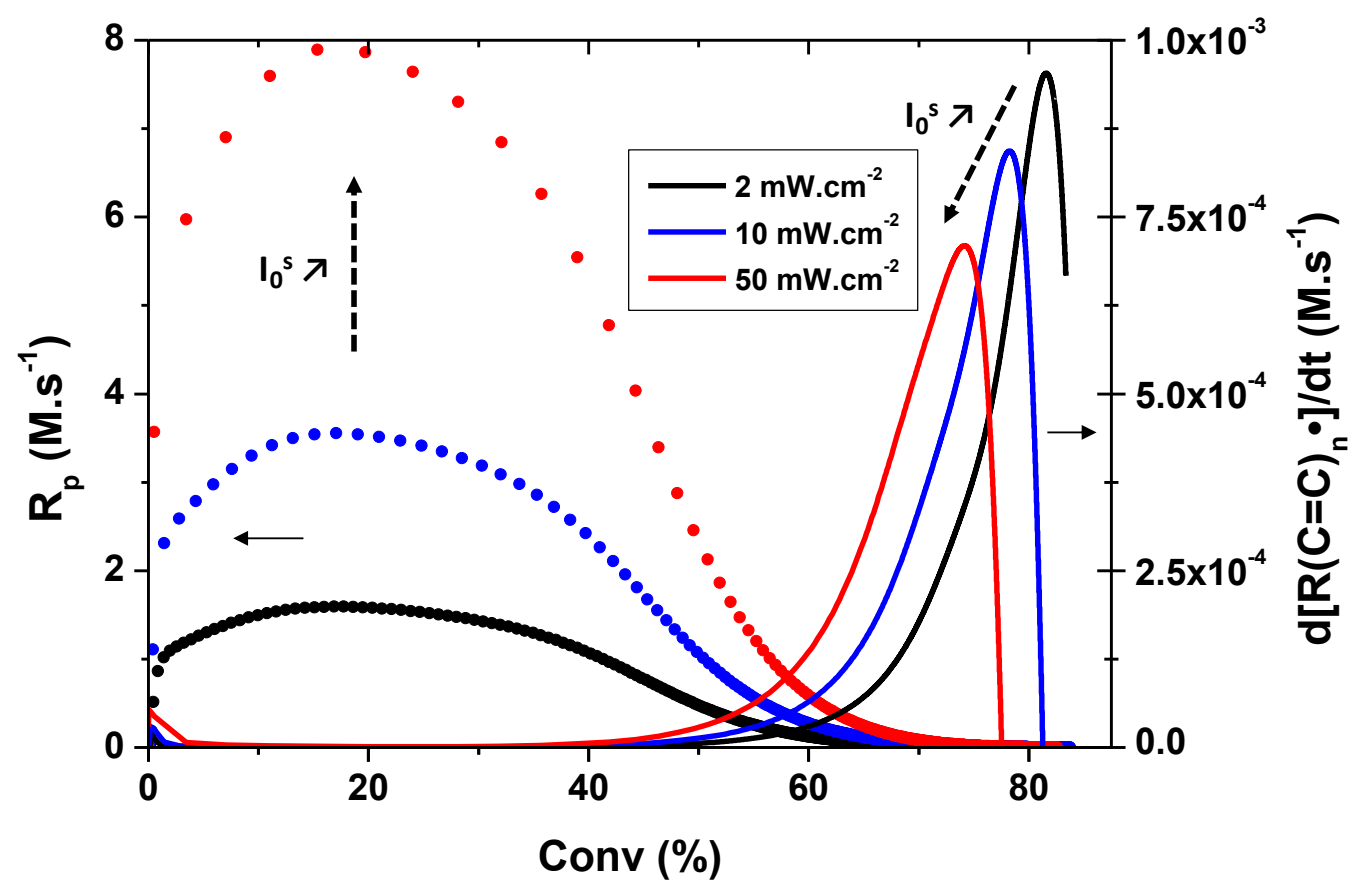

Figure 12: Evolution of the rate of polymerization $R_{p}$ (circles, left) and the rate of

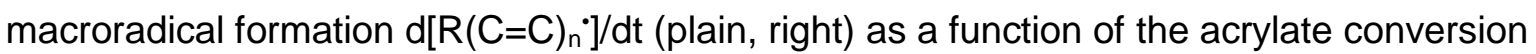
for different incident light intensities ( $1 \mathrm{wt} \%$ TPO, $\lambda_{\text {irr }}=395 \mathrm{~nm}$ ). The horizontal arrows indicate the corresponding axis.

Maximal values of $R_{p}$ curves are obtained for the same conversion, which is consistent with autoacceleration/autodeceleration processes starting at a certain degree of medium vitrification. The evolution of $d\left[R(C=C)_{n}{ }^{\circ}\right] / d t$ is also interesting: its value is almost equal to zero 
from 5 to $40 \%$ of conversion, strongly increases and finally drops off at higher conversions. This increase could be explained by the diffusional control of both the propagation and bimolecular termination steps (vide supra). Indeed, the macroradical concentration strongly increases when bimolecular termination is reduced. The final decrease is associated to the progressive photolysis of TPO and the associated cessation of initiation. As a consequence, macroradicals are not produced but termination reactions take place. Figure 12 then reveals that the steady-state assumption should be valid between 5 and $40 \%$ of conversion, but not for higher values. Indeed, the macroradical concentration strongly evolves in this range. It confirms previous observations made in the literature with a simpler kinetic model [78] and then reconsiders the validity of the steady-state assumption still generally applied on $\left[R(C=C)_{n}{ }^{*}\right]$ in photopolymerization kinetics.

\subsubsection{Influence of initial photoinitiator concentration on termination reactions}

The comparison of experimental and simulated data for 0.5 and 1 wt\% TPO in Figure 2 proves the ability of the model to account for the effect of the initial TPO concentration on the kinetics. Figure 13 represents the results obtained for [TPO]o values comprised between $0.5 \times 10^{-2}$ and $10 \times 10^{-2} \mathrm{M}(0.15$ to $3 \mathrm{wt} \%)$. Contrary to the effect of $10^{\mathrm{s}}$, the final conversion increases when [TPO]o is enhanced. However, the rate of the FRP process is once again increased and the inhibition time reduced, as expected from the literature. The effects of increasing [TPO $]_{0}$ on $R_{p}$ curves are similar to the ones obtained for the increase of $I_{0}$ s: $i$. the inhibition time is reduced, ii. the $R_{p}{ }^{\max }$ position is moved to shorter times, iii. the $\mathrm{R}_{\mathrm{p}}{ }^{\max }$ values are greater, iv. the curves are sharper (both faster autoacceleration and autodeceleration). 


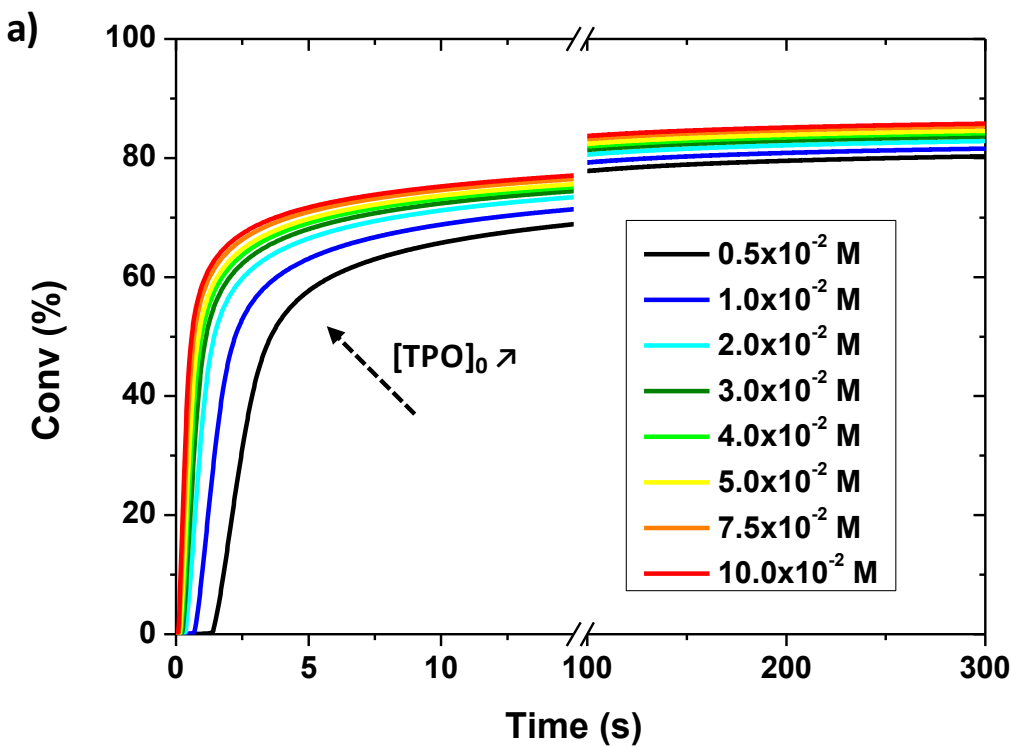

b)

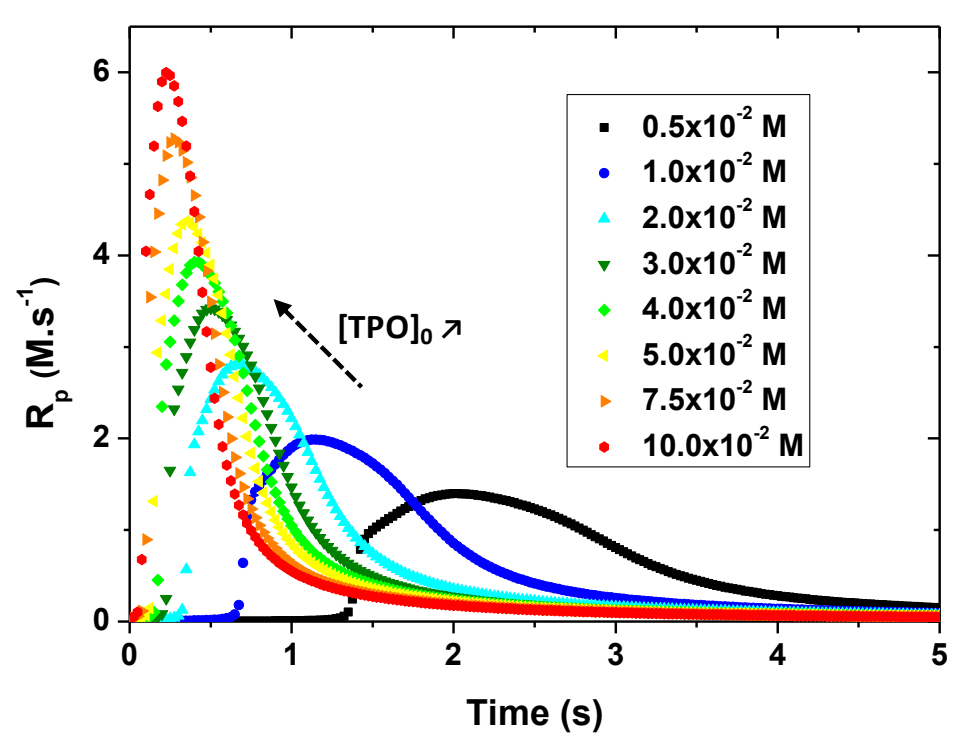

Figure 13: Effect of the TPO initial concentration on the simulated a) conversion b) rate of polymerization $\left(\lambda_{\text {irr }}=395 \mathrm{~nm}, 10 \mathrm{~mW} \cdot \mathrm{cm}^{-2}\right)$.

Increasing the initial concentration of TPO enhances the amount of primary radical produced per second, by means of a higher absorbance of the sample. Macroradicals are then formed faster (see Figure S13 in the ESI) and the total consumption of the initially dissolved $\mathrm{O}_{2}$ molecules occurs earlier, reducing the inhibition time. As for $\mathrm{I}_{0} \mathrm{~s}$, the vitrification of the polymer network would occur faster, so both autoacceleration and autodeceleration processes are faster. Finally, maximal $R_{p}$ values increase with [TPO] $]_{0}$ because of the faster rate of primary radical and macroradical production. The effect on the final conversion comes from an enhanced macroradical formation combined to a decrease of the bimolecular termination proportion when [TPO] $]_{0}$ is raised (vide infra). 
Figure 14 represents the evolution of the termination fractions at final conversion as a function of the initial TPO concentration. It can be seen that macroradical bimolecular termination decreases with [TPO]o, while radical trapping by occlusion and PRT are enhanced. These results can be explained with Figure 15, which represents the evolution of the termination and macroradical fractions and that of the TPO concentration as a function of the polymer conversion for three initial [TPO] $]_{0}$ values.

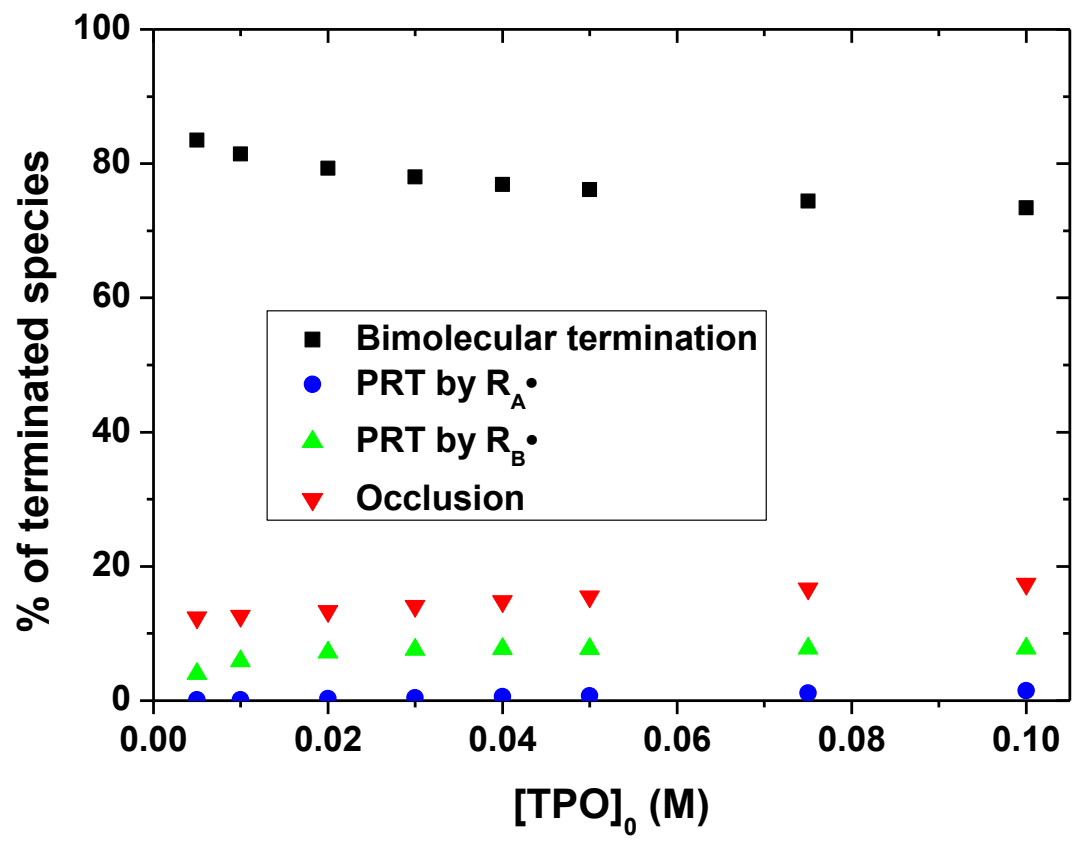

Figure 14: Influence of the initial TPO concentration on the termination modes at final conversion $\left(\lambda_{\text {irr }}=395 \mathrm{~nm}, 10 \mathrm{~mW} \cdot \mathrm{cm}^{-2}\right)$. 


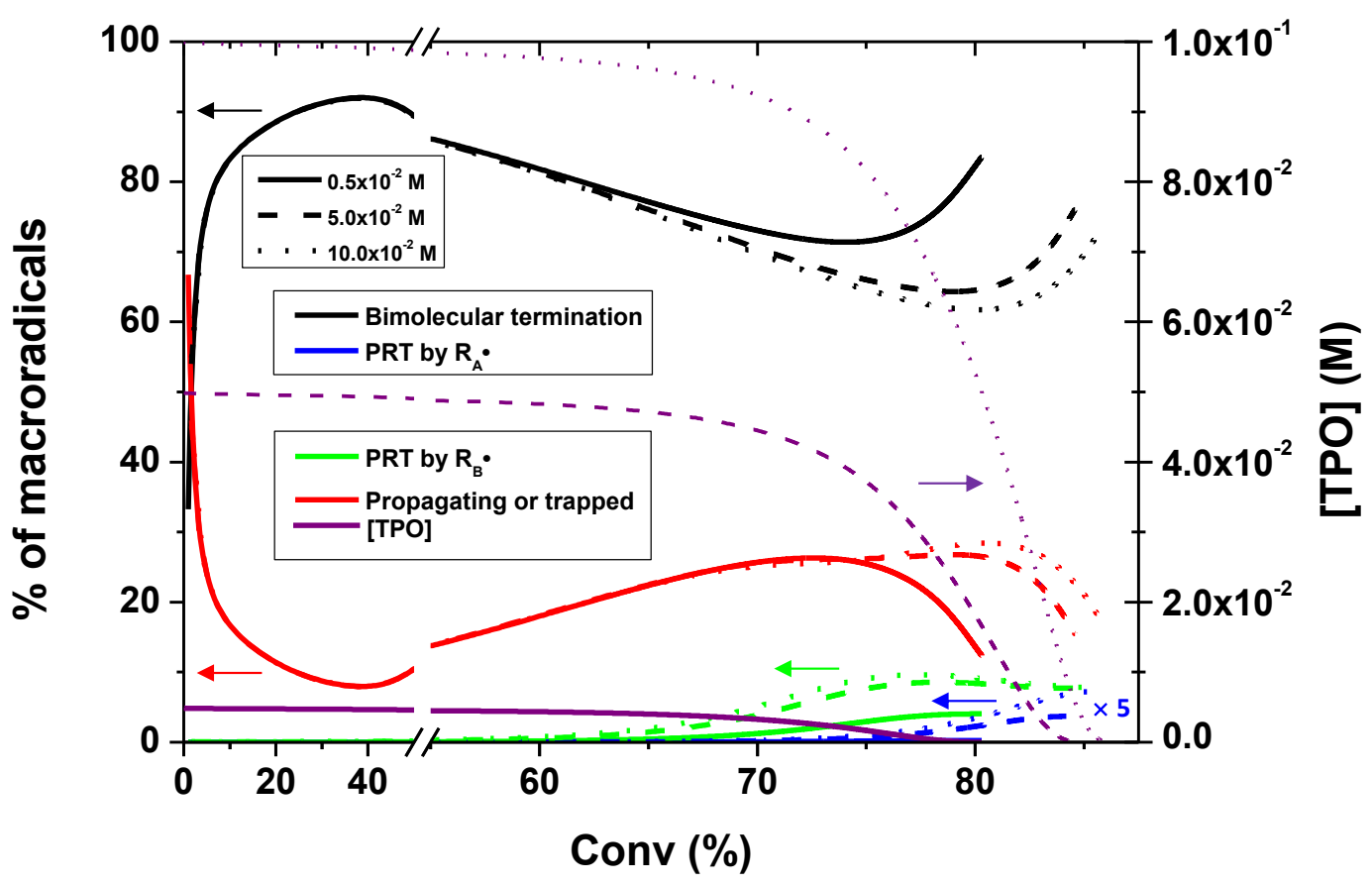

Figure 15: Change in the fractions of terminated species and propagating or trapped macroradicals (left) and of the TPO concentration (right) for $0.5 \times 10^{-2}$ (solid), $5 \times 10^{-2}$ (dashed) and $10 \times 10^{-2} \mathrm{M}$ (dotted) of TPO as a function of the acrylate conversion $\left(\lambda_{\text {irr }}=395 \mathrm{~nm}, 10\right.$ $\left.\mathrm{mW} . \mathrm{cm}^{-2}\right)$. The horizontal arrows indicate the corresponding axis - blue curve is magnified by a factor 5 for clarity.

It can be seen that the successive steps for the change in the termination and macroradical fractions are not identical to what was observed when the incident light intensity is increased, meaning that the $[\mathrm{TPO}]_{0}$ value has different effects on the final increase of bimolecular termination and the decrease of macroradical fractions, as well as the onset of PRT. Indeed, as the TPO initial concentration is increased, the proportion of bimolecular termination decreases faster and to a lower value. Moreover, its final increase is delayed to higher conversions. In parallel, the macroradical fraction increases while PRT becomes significant earlier and represents a higher proportion of termination reactions when [TPO $]_{0}$ is increased. Finally, TPO is consumed slowly when its initial concentration is raised.

The previous sections of this work revealed that the final increase of bimolecular termination and the decrease of macroradical fraction are directly linked to the disappearance of TPO. The delayed final increase of the bimolecular termination proportion and the decrease of the macroradical fraction observed in Figure 15 are then consistent with the slower photolysis of the photoinitiator when $[\mathrm{TPO}]_{0}$ is raised. Indeed, the macroradical production ceases at a higher conversion and bimolecular termination has less time to occur between the remaining 
macroradicals, which explains the higher final fraction of macroradicals. The earlier efficiency of PRT could be explained by the higher concentration of primary radicals and their increased formation rate when [TPO] $]_{0}$ is raised. Indeed, the $R_{t, P R T} / R_{i}$ ratio is higher for a same conversion when [TPO] increases, promoting PRT over initiation at a lower conversion (see Figure S14 in the $\mathrm{ESI}$ ). As a consequence, this termination way finally represents a higher proportion of the macroradicals produced.

\section{Conclusion}

A kinetic model taking into account simultaneously all the possible termination pathways (bimolecular termination, primary radical termination and radical trapping by occlusion) was successfully applied to the photopolymerization kinetics initiated by a type-I photoinitiator. Comparison with experiments reveals a good agreement both on conversion and rate of polymerization curves. The inhibition by the initially dissolved oxygen molecules in laminated systems was considered in order to take into account the experimental inhibition time. It points out for the first time that the macroradicals act as the principal $\mathrm{O}_{2}$ scavengers at the early stages of the process, whereas the primary initiating radicals are almost totally involved in initiation reactions with the monomer.

The model was then adapted to identify the relative contribution of the different termination ways throughout the photopolymerization process with numerical values. It was shown that bimolecular termination remains the major termination reaction during the whole process. However, its ratio, as well as that of primary radical termination and macroradicals, actually evolves because of: $i$. the progressive diffusion control of the polymerization reactions as the polymer network grows, ii. the cessation of initiation when the photoinitiator is totally consumed. The impact of the incident light intensity and the initial photoinitiator concentration on the termination modes was also investigated. As expected, the photopolymerization process is faster and the inhibition time lower when both parameters are increased, but the final conversion only increases when [TPO $]_{0}$ is raised. Increasing the incident light intensity enhances the fraction of bimolecular termination and that of PRT. However, it is interesting to notice that the former remains the major termination pathway even at high incident intensity. When the initial photoinitiator concentration is raised, PRT becomes more efficient, but radical trapping by occlusion in the glassy 3D polymer network also surprisingly increases.

It will be very interesting to extend this approach to more complex photoinitiating systems (type-Il or three-component photocyclic ones), as the underlying photochemistry will be impacted by the formation of the polymer network during the irradiation but will also influence back the polymerization process. This will be the subject of a forthcoming paper. 


\section{Declaration of interest}

The authors declare that they have no competing financial interests.

\section{Funding}

ANR and Mäder are fully acknowledged for financial support of the DeepCure project \#ANR13-CHIN-0004-01.

\section{Appendix A. Supplementary material}

Supplementary data associated with this article can be found, in the online version, at XX.

\section{References}

[1] in J-P. Fouassier, J.F. Rabek (Eds.), Radiation Curing in Polymer Science and Technology Volume I: Fundamentals and Methods, Elsevier, London, 1993.

[2] J-P. Fouassier, Photoinitiation, Photopolymerization, and Photocuring: Fundamentals and Applications, Hanser, Munich, 1995.

[3] C. Decker, Photoinitiated crosslinking polymerization, Prog. Polym. Sci. 21 (1996) 593650.

[4] Y. Yagci, S. Jockusch, N.J. Turro, Photoinitiated polymerization: advances, challenges, and opportunities, Macromolecules 43 (2010) 6245-6260.

[5] P. Nesvadba, Radical polymerization in industry, in: C. Chatgilialoglu, A. Studer (Eds.), Encyclopedia of Radicals in Chemistry, Biology, and Materials, Wiley, Hoboken NJ, 2012, pp. 1-36.

[6] G. Odian, Principles of Polymerization, fourth ed., Wiley, Hoboken NJ, 2004.

[7] Y. Gnanou, M. Fontanille, Organic and Physical Chemistry of Polymers, Wiley, Hoboken NJ, 2008.

[8] E. Andrzejewska, Photopolymerization kinetics of multifunctional monomers, Prog. Polym. Sci. 26 (2001) 605-665.

[9] A. Ibrahim, V. Maurin, C. Ley, X. Allonas, C. Croutxé-Barghorn, F. Jasinski, Investigation of termination reactions in free radical photopolymerization of UV powder formulations, Eur. Polym. J. 48 (2012) 1475-1484. 
[10] J-P. Fouassier, J. Lalevée, Photoinitiators for Polymer Synthesis: Scope, Reactivity, and Efficiency, Wiley, Weinheim, 2013.

[11] W.A. Green, Industrial Photoinitiators: A Technical Guide, CRC Press, Boca Raton, 2010.

[12] J-P. Fouassier, X. Allonas, J. Lalevée, C. Dietlin, Photoinitiators for free radical polymerization reactions, in: N.S. Allen (Ed.), Photochemistry and Photophysics of Polymer Materials, Wiley, Hoboken NJ, 2010, pp. 351-419.

[13] A. Ibrahim, L.H. Di Stefano, O. Tarzi, H. Tar, C. Ley, X. Allonas, High-performance photoinitiating systems for free radical photopolymerization. Application to holographic recording, Photochemistry and Photobiology 89 (2013) 1283-1290.

[14] M.D. Goodner, C.N. Bowman, Modeling primary radical termination and its effects on autoacceleration in photopolymerization kinetics, Macromolecules 32 (1999) 6552-6559.

[15] G. David, J-J. Robin, B. Boutevin, Synthesis of carboxy-telechelic oligostyrene by deadend polymerization: evaluation of primary radical termination by kinetic study and kinetic simulation model, J. Polym. Sci. Pol. Chem. 39 (2001) 2740-2750.

[16] J.V. Kelly, M.R. Gleeson, C.E. Close, F.T. O'Neill, J.T. Sheridan, S. Gallego, C. Neipp, Temporal and non-ideal behavior in photopolymers, Proc. SPIE 5827 (2005) 95-106.

[17] J.V. Kelly, F.T. O’Neill, J.T. Sheridan, C. Neipp, S. Gallego, M. Ortuno, Holographic photopolymer materials: nonlocal polymerization-driven diffusion under nonideal kinetic conditions, J. Opt. Soc. Am. B 22 (2005) 407-416.

[18] G.K. Fraenkel, J.M. Hirshon, C. Walling, Detection of polymerization radicals by paramagnetic resonance, J. Am. Chem. Soc. 76 (1954) 3606-3606.

[19] J.G. Kloosterboer, G.F.C.M. Lijten, F.J.A.M. Greidanus, Structure and stability of polyacrylate radicals trapped in a network, Polym. Commun. 27 (1986) 268-271.

[20] C. Decker, K. Moussa, Radical trapping in photopolymerized acrylic networks, J. Polym. Sci. Pol. Chem. 25 (1987) 739-742.

[21] I.R. Bellobono, E. Selli, L. Righetto, P. Rafellini, L. Trevisan, Kinetic effect of radiation intensity and thermal after-treatment in the photopolymerization of diallyl oxydiethylene dicarbonate, Makromol. Chem. 190 (1989) 1945-1952. 
[22] M.E. Best, P.H. Kasai, Electron spin resonance study of radicals in photopolymerized di(meth)acrylate network, Macromolecules 22 (1989) 2622-2627.

[23] S. Zhu, Y. Tian, A.E. Hamielec, D.R. Eaton, Termination of trapped radicals at elevated temperatures during copolymerization of MMA/EGDMA, Polymer 31 (1990) 1726-1734.

[24] S. Zhu, Y. Tian, A.E. Hamielec, D.R. Eaton, Radical trapping and termination in freeradical polymerization of methyl methacrylate, Macromolecules 23 (1990) 1144-1150.

[25] in J-P. Fouassier, J.F. Rabek (Eds.), Radiation Curing in Polymer Science and Technology Volume III: Polymerization Mechanism, Elsevier, London, 1993.

[26] K.S. Anseth, K.J. Anderson, C.N. Bowman, Radical concentrations, environments, and reactivities during crosslinking polymerizations, Macromol. Chem. Phys. 197 (1996) 833848.

[27] K.A. Berchtold, T.W. Randolph, C.N. Bowman, Propagation and termination kinetics of cross-linking photopolymerizations studied using electron paramagnetic resonance spectroscopy in conjunction with near IR spectroscopy, Macromolecules 38 (2005) 69546964.

[28] M. Wen, A.V. McCormick, A kinetic model for radical trapping in photopolymerization of multifunctional monomers, Macromolecules 33 (2000) 9247-9254.

[29] Y. Zhang, D.E. Kranbuehl, H. Sautereau, G. Seytre, J. Dupuy, Study of UV cure kinetics resulting from a changing concentration of mobile and trapped radicals, Macromolecules 41 (2008) 708-715.

[30] Y. Zhang, D.E. Kranbuehl, H. Sautereau, G. Seytre, J. Dupuy, Modeling and measuring UV cure kinetics of thick dimethacrylate samples, Macromolecules 42 (2009) 203-210.

[31] in J-P. Fouassier, J.F. Rabek (Eds.), Radiation Curing in Polymer Science and Technology Volume II: Photoinitiating Systems, Elsevier, London, 1993.

[32] R. Pynaert, J. Buguet, C. Croutxé-Barghorn, P. Moireau, X. Allonas, Effect of reactive oxygen species on the kinetics of free radical photopolymerization, Polym. Chem. 4 (2013) 2475-2479.

[33] S.C. Ligon, B. Husár, H. Wutzel, R. Holman, R. Liska, Strategies to reduce oxygen inhibition in photoinduced polymerization, Chem. Rev. 114 (2014) 557-589. 
[34] M. Gleeson, J.V. Kelly, C.E. Close, F.T. O'Neill, J.T. Sheridan, Effects of absorption and inhibition during grating formation in photopolymer materials, J. Opt. Soc. Am. B 23 (2006) 2079-2088.

[35] M.R. Gleeson, J.V. Kelly, D. Sabol, C.E. Close, S. Liu, J.T. Sheridan, Modeling the photochemical effects present during holographic grating formation in photopolymer materials, J. Appl. Phys. 102 (2007) 023108-1-023108-9.

[36] E. Von Trommsdorff, H. Köhle, P. Lagally, Zur Polymerisation des Methacrylsäuremethylesters, Makromol. Chem. 1 (1948) 169-198.

[37] G.V. Schulz, Über die Polymerisationskinetik in Hochkonzentrierten Systemen, Z. Phys. Chem. 8 (1956) 290-317.

[38] S.K. Soh, D.C. Sundberg, Diffusion-controlled vinyl polymerization. II. Limitations on the gel effect, J. Polym. Sci. Pol. Chem. Ed. 20 (1982) 1315-1329.

[39] M. Stickler, Free-radical polymerization kinetics of methyl methacrylate at very high conversions, Makromol. Chem. 184 (1983) 2563-2579.

[40] M.J. Ballard, D.H. Napper, R.G. Gilbert, D.F. Sangster, Termination-rate coefficients in methyl methacrylate polymerizations, J. Polym. Sci. Pol. Chem. 24 (1986) 1027-1041.

[41] M. Buback, B. Degener, B. Huckestein, Conversion dependence of free-radical polymerization rate coefficients from laser-induced experiments, 1. Butyl acrylate, Makromol. Chem. Rapid. Commun. 10 (1989) 311-316.

[42] M. Buback, Free-radical polymerization kinetics in extended ranges of temperature, pressure and monomer conversion, Macromol. Symp. 111 (1996) 229-242.

[43] M. Buback, Free-radical polymerization up to high conversion. A general kinetic treatment, Makromol. Chem. 191 (1990) 1575-1587.

[44] M. Buback, B. Huckestein, G.T. Russell, Modeling of termination in intermediate and high conversion free radical polymerizations, Macromol. Chem. Phys. 195 (1994) 539-554.

[45] W.D. Cook, Photopolymerization kinetics of oligo(ethylene oxide) and oligo(methylene) oxide dimethacrylates, J. Polym. Sci. Pol. Chem. 31 (1993) 1053-1067.

[46] K.S. Anseth, C.N. Bowman, Reaction diffusion enhanced termination in polymerizations of multifunctional monomers, Polym. Reac. Eng. 1 (1993) 499-520. 
[47] K.A. Berchtold, T.M. Lovestead, C.N. Bowman, Coupling chain length dependent and reaction diffusion controlled termination in the free radical polymerization of multivinyl (meth)acrylates, Macromolecules 35 (2002) 7968-7975.

[48] K.S. Anseth, C.M. Wang, C.N. Bowman, Reaction behaviour and kinetic constants for photopolymerizations of multi(meth)acrylate monomers, Polymer 35 (1994) 3243-3250.

[49] K.S. Anseth, C.N. Bowman, N.A. Peppas, Polymerization kinetics and volume relaxation behavior of photopolymerized multifunctional monomers producing highly crosslinked networks, J. Polym. Sci. Pol. Chem. 32 (1994) 139-147.

[50] K.S. Anseth, C.M. Wang, C.N. Bowman, Kinetic evidence of reaction diffusion during the polymerization of multi(meth)acrylate monomers, Macromolecules 27 (1994) 650-655.

[51] K.S. Anseth, L.M. Kline, T.A. Walker, K.J. Anderson, C.N. Bowman, Reaction kinetics and volume relaxation during polymerizations of multiethylene glycol dimethacrylates, Macromolecules 28 (1995) 2491-2499.

[52] J.S. Young, C.N. Bowman, Effect of polymerization temperature and cross-linker concentration on reaction diffusion controlled termination, Macromolecules 32 (1999) 6073-6081.

[53] T.J. White, W.B. Liechty, C.A. Guymon, The influence of N-vinyl pyrrolidone on polymerization kinetics and thermo-mechanical properties of crosslinked acrylate polymers, J. Polym. Sci. Pol. Chem. 45 (2007) 4062-4073.

[54] P.J. Flory, Molecular size distribution in three dimensional polymers. I. Gelation, J. Am. Chem. Soc. 63 (1941) 3083-3090.

[55] P.J. Flory, Molecular size distribution in three dimensional polymers. II. Trifunctional branching units, J. Am. Chem. Soc. 63 (1941) 3091-3096.

[56] P.J. Flory, Molecular size distribution in three dimensional polymers. III. Tetrafunctional branching units, J. Am. Chem. Soc. 63 (1941) 3096-3100.

[57] P.J. Flory, Statistical Mechanics of Chain Molecules, Intersciences Publishers, New York, 1969.

[58] P. Manneville, L. de Seze, Percolation and gelation by additive polymerization, in: J. Della Dora, J. Demongeot, B. Lacolle (Eds.), Numerical Methods in the Study of Critical Phenomena (Springer Series in Synergetics Vol. 9), Springer, Berlin, 1981, pp. 116-124. 
[59] U.P. Schröder, W. Oppermann, Computer simulation of network formation via crosslinking copolymerization, Macromol. Theory Simul. 6 (1997) 151-160.

[60] M. Ghiass, A.D. Rey, B. Dabir, Simulation of non-linear free-radical polymerization using a percolation kinetic gelation model, Macromol. Theory Simul. 10 (2001) 657-667.

[61] M. Wen, L.E. Scriven, A.V. McCormick, Kinetic gelation modeling: structural inhomogeneity during cross-linking polymerization, Macromolecules 36 (2003) 41404150 .

[62] M. Wen, L.E. Scriven, A.V. McCormick, Kinetic gelation modeling: kinetics of crosslinking polymerization, Macromolecules 36 (2003) 4151-4159.

[63] D.S. Achilias, A review of modeling of diffusion controlled polymerization reactions, Macromol. Theory Simul. 16 (2007) 319-347.

[64] L. Lecamp, P. Lebaudy, B. Youssef, C. Bunel, Simulation of temperature distributions within monomer film during photopolymerization, J. Therm. Anal. Calorim. 51 (1998) 889896.

[65] L. Lecamp, P. Lebaudy, B. Youssef, C. Bunel, Simulation of conversion profiles and temperature distributions within dimethacrylate thick material during photopolymerization, Macromol. Symp. 148 (1999) 77-86.

[66] L. Lecamp, P. Lebaudy, B. Youssef, C. Bunel, Influence of UV radiation wavelength on conversion and temperature distribution profiles within dimethacrylate thick material during photopolymerization, Polymer 42 (2001) 8541-8547.

[67] P. Kerbouc'h, P. Lebaudy, L. Lecamp, C. Bunel, Numerical simulation to correlate photopolymerization kinetics monitoring by RT-NIR spectroscopy and photocalorimetry, Thermochim. Acta 410 (2004) 73-78.

[68] N. Désilles, L. Lecamp, P. Lebaudy, B. Youssef, Z. Lebaudy, C. Bunel, Simulation of conversion profiles within dimethacrylate thick material during photopolymerization. Validation of the simulation by thermal analysis data. Application to the synthesis of a gradient structure material, Polymer 47 (2006) 193-199.

[69] V. Azan, L. Lecamp, P. Lebaudy, C. Bunel, Simulation of the photopolymerization gradient inside a pigmented coating, Prog. Org. Coat. 58 (2007) 70-75. 
[70] S. Bayou, M. Mouzali, F. Aloui, L. Lecamp, P. Lebaudy, Simulation of conversion profiles inside a thick dental material photopolymerized in the presence of nanofillers, Polym. J. 45 (2013) 863-870.

[71] G. Terrones, A.J. Pearlstein, Effects of optical attenuation and consumption of a photobleaching initiator on local initiation rates in photopolymerizations, Macromolecules 34 (2001) 3195-3204.

[72] G. Terrones, A.J. Pearlstein, Effects of kinetics and optical attenuation on the completeness, uniformity, and dynamics of monomer conversion in free-radical photopolymerizations, Macromolecules 34 (2001) 8894-8906.

[73] G. Terrones, A.J. Pearlstein, Nonuniformity of chain-length distributions in photopolymerized layers, Macromolecules 36 (2003) 6346-6358.

[74] G. Terrones, A.J. Pearlstein, Diffusion-induced nonuniformity of photoinitiation in a photobleaching medium, Macromolecules 37 (2004) 1565-1575.

[75] M.R. Gleeson, J.T. Sheridan, A review of the modelling of free-radical photopolymerization in the formation of holographic gratings, J. Opt. A: Pure Appl. Opt. 11 (2009) 024008-1-024008-12.

[76] H. Li, Y. Qi, J. Guo, J.T. Sheridan, Analysis of the absorptive behavior of photopolymer materials. Part I. Theoretical modeling, J. Mod. Opt. 62 (2015) 143-154.

[77] H. Li, Y. Qi, E. Tolstik, J. Guo, J.T. Sheridan, Analysis of the absorptive behavior of photopolymer materials. Part II. Experimental validation, J. Mod. Opt. 62 (2015) 155-165.

[78] C.N. Bowman, N.A. Peppas, Coupling of kinetics and volume relaxation during polymerizations of multiacrylates and multimethacrylates, Macromolecules 24 (1991) 1914-1920.

[79] M.D. Goodner, H.R. Lee, C.N. Bowman, Method for determining the kinetic parameters in diffusion-controlled free-radical homopolymerizations, Ind. Eng. Chem. Res. 36 (1997) 1247-1252.

[80] M.D. Goodner, C.N. Bowman, Development of a comprehensive free radical photopolymerization model incorporating heat and mass transfer effects in thick films, Chem. Eng. Sci. 57 (2002) 887-900.

[81] T.M. Lovestead, A.K. O'Brien, C.N. Bowman, Models of multivinyl free radical photopolymerization kinetics, J. Photochem. Photobiol. A 159 (2003) 135-143. 
[82] K. Taki, Y. Watanabe, H. Ito, M. Ohshima, Effect of oxygen inhibition on the kinetic constants of the UV-radical photopolymerization of diurethane dimethacrylate/photoinitiator systems, Macromolecules 47(2014) 1906-1913.

[83] K. Taki, Y. Watanabe, T. Tanabe, H. Ito, M. Ohshima, Oxygen concentration and conversion distributions in a layer-by-layer UV-cured film used as a simplified model of a 3D UV inkjet printing system, Chem. Eng. Sci. 158 (2017) 569-579.

[84] J. Christmann, X. Allonas, C. Ley, A. Ibrahim, C. Croutxé-Barghorn, Triazine-based typeII photoinitiating system for free radical photopolymerization: mechanism, efficiency, and modeling, Macromol. Chem. Phys. 218 (2017) 1600597-1-1600597-10.

[85] C. Decker, K. Moussa, Real-time kinetic study of laser-induced polymerization, Macromolecules 22 (1989) 4455-4462.

[86] T. Sumiyoshi, W. Schnabel, On the reactivity of phosphonyl radicals towards olefinic compounds, Makromol. Chem. 186 (1985) 1811-1823.

[87] T. Sumiyoshi, W. Schnabel, A. Henne, P. Lechtken, On the photolysis of acylphosphine oxides: 1. Laser flash photolysis studies with 2,4,6-trimethylbenzoyldiphenylphosphine oxide, Polymer 26 (1985) 141-146.

[88] G.W. Sluggett, P.F. McGarry,, I.V. Koptyug, N.J. Turro, , Laser flash photolysis and timeresolved ESR study of phosphinoyl radical structure and reactivity, J. Am. Chem. Soc. 118 (1996) 7367-7372.

[89] S. Jockusch, N.J. Turro, Phosphinoyl radicals: structure and reactivity. A laser flash photolysis and time-resolved ESR investigation, J. Am. Chem. Soc. 120 (1998) 1177311777.

[90] I. Gatlik, P. Rzadek, G. Gescheidt, G. Rist, B. Hellrung, J. Wirz, K. Dietliker, G. Hug, M. Kunz, J-P. Wolf, Structure-reactivity relationships in radical reactions: a novel method for the simultaneous determination of absolute rate constants and structural features, J. Am. Chem. Soc. 121 (1999) 8332-8336.

[91] M. Weber, I.V. Khudyakov, N.J. Turro, Electron spin resonance and laser flash photolysis study of radical addition to vinyl acrylate and related alkenes, J. Phys. Chem. A 106 (2002) 1938-1945. 
[92] M. Weber, N.J. Turro, A novel approach for measuring absolute rate constants by pulsed electron spin resonance: addition of phosphinoyl and 2-hydroxy-2-propyl radicals to several alkenes, J. Phys. Chem. A 107 (2003) 3326-3334.

[93] H. Takahashi, Y. Marushima, K. Tsuji, K. Shibuya, A. Kawai, Addition rate constants of phosphorus- and carbon-centered radicals to double bond of monomers as studied by a pulsed electron paramagnetic resonance method, J. Phys. Chem. A 119 (2015) 82618268.

[94] C.S. Colley, D.C. Grills, N.A. Besley, S. Jockusch, P. Matousek, A.W. Parker, M. Towrie, N.J. Turro, P.M.W. Gill, M.W. George, Probing the reactivity of photoinitiators for free radical polymerization: time-resolved infrared spectroscopic study of benzoyl radicals, J. Am. Chem. Soc. 124 (2002) 14952-1958.

[95] X. Allonas, J. Lalevée, J-P. Fouassier, Investigation of cleavage processes in photoinitiators: from experiments to molecular modeling, J. Photochem. Photobiol. A 159 (2003) 127-133.

[96] T. Scherzer, H. Langguth, The effect of temperature on the induction period in the photoinitiated polymerization of tripropylene glycol diacrylate, Nucl. Instr. and Meth. in Phys. Res. B 185 (2001) 276-282.

[97] M.D. Goodner, C.N. Bowman, Modeling and experimental investigation of light intensity and initiator effects on solvent-free photopolymerizations, in: T.E. Long, M.O. Hunt (Eds.), Solvent-Free Polymerization and Processes (ACS Symposium Series Vol. 713), American Chemical Society, Washington DC, 1999, pp. 220-231.

[98] M.D. Goodner, C.N. Bowman, Modeling primary radical termination and its effects on autoacceleration in photopolymerization kinetics, Macromolecules 32 (1999) 6552-6559. 